\title{
Lifetimes of doubly heavy baryons $\mathcal{B}_{b b}$ and $\mathcal{B}_{b c}$
}

\author{
Hai-Yang Cheng ${ }^{1}$ and Fanrong $\mathrm{Xu}^{2}$ \\ ${ }^{1}$ Institute of Physics, Academia Sinica, Taipei, Taiwan 115, Republic of China \\ ${ }^{2}$ Department of Physics, Jinan University, Guangzhou 510632, People's Republic of China
}

(Received 21 March 2019; published 16 April 2019)

\begin{abstract}
Lifetimes of the doubly heavy baryons $\mathcal{B}_{b b}$ and $\mathcal{B}_{b c}$ are analyzed within the framework of the heavy quark expansion (HQE). Lifetime differences arise from the spectator effects such as $W$-exchange and Pauli interference. For doubly bottom baryons, the lifetime pattern is $\tau\left(\Omega_{b b}^{-}\right) \sim \tau\left(\Xi_{b b}^{-}\right)>\tau\left(\Xi_{b b}^{0}\right)$. The $\Xi_{b b}^{0}$ baryon is shortest-lived owing to the $W$-exchange contribution, while $\Xi_{b b}^{-}$and $\Omega_{b b}^{-}$have similar lifetimes as they both receive contributions from destructive Pauli interference. We find the lifetime ratio $\tau\left(\Xi_{b b}^{-}\right) / \tau\left(\Xi_{b b}^{0}\right)=1.26$. The large $W$-exchange contribution to $\Xi_{b c}^{0}$ through the subprocess $c d \rightarrow u s \rightarrow c d$ and the sizable destructive Pauli interference contribution to $\Xi_{b c}^{+}$imply a substantial lifetime difference between $\Xi_{b c}^{+}$and $\Xi_{b c}^{0}$. In the presence of subleading $1 / m_{c}$ and $1 / m_{b}$ corrections to the spectator effects, we find that $\tau\left(\Omega_{b c}^{0}\right)$ becomes longest-lived. This is because $\Gamma_{+}^{\text {int }}$ and $\Gamma^{\text {semi }}$ for $\Omega_{b c}^{0}$ are subject to large cancellation between dimension- 6 and -7 operators. This implies that the subleading corrections are too large to justify the validity of the HQE. Demanding that $\Gamma_{\text {int }}^{c s}\left(\Omega_{b c}^{0}\right), \Gamma_{\text {int }}^{\mathrm{SL}, c s}\left(\Omega_{b c}^{0}\right)$ be positive and $\Gamma_{\text {int- }}^{c u}\left(\Xi_{b c}^{+}\right)$be negative, we conjecture that $1.68 \times 10^{-13} s<\tau\left(\Omega_{b c}^{0}\right)<3.70 \times 10^{-13} \mathrm{~s}, 4.09 \times 10^{-13} s<\tau\left(\Xi_{b c}^{+}\right)<6.07 \times 10^{-13}$ s, and $0.93 \times 10^{-13} s<\tau\left(\Xi_{b c}^{0}\right)<$ $1.18 \times 10^{-13} \mathrm{~s}$. Hence, the lifetime hierarchy of $\mathcal{B}_{b c}$ baryons is expected to be $\tau\left(\Xi_{b c}^{+}\right)>\tau\left(\Omega_{b c}^{0}\right)>\tau\left(\Xi_{b c}^{0}\right)$.
\end{abstract}

DOI: 10.1103/PhysRevD.99.073006

\section{INTRODUCTION}

After the discovery of the doubly charmed baryon $\Xi_{c c}^{++}$ in the $\Lambda_{c}^{+} K^{-} \pi^{+} \pi^{+}$mass spectrum [1], LHCb proceeded to measure its lifetime [2]:

$$
\tau\left(\Xi_{c c}^{++}\right)=\left(2.56_{-0.22}^{+0.24} \pm 0.14\right) \times 10^{-13} s .
$$

The theoretical predictions of doubly charmed baryon lifetimes in the literature [3-9] listed in Table I spread a large range, especially for $\Xi_{c c}^{++}$. It appears that the early predictions of $\tau\left(\Xi_{c c}^{++}\right)$were too large compared to the experiment. The lifetime pattern is expected to be $\tau\left(\Xi_{c c}^{++}\right)>\tau\left(\Omega_{c c}^{+}\right)>\tau\left(\Xi_{c c}^{+}\right)$.

In this work, we would like to generalize our previous study of doubly charmed baryon lifetimes [8] to the doubly bottom baryons $\mathcal{B}_{b b}$ and charm-bottom baryons $\mathcal{B}_{b c}$. Some predictions available in the literature are shown in Table II. It is well known that the lifetime differences stem mainly from the spectator effects such as weak annihilation and Pauli interference. Spectator effects are depicted in Figs. 1 and 2 for $\mathcal{B}_{b b}$ and $\mathcal{B}_{b c}$ baryons, respectively. Calculations in

Published by the American Physical Society under the terms of the Creative Commons Attribution 4.0 International license. Further distribution of this work must maintain attribution to the author(s) and the published article's title, journal citation, and DOI. Funded by SCOAP ${ }^{3}$.
$[5,7,9,10]$ indicate that the lifetimes of $\Xi_{b b}^{0}$ and $\Xi_{b b}^{-}$are close to each other. However, we see from Fig. 1 that $\Xi_{b b}^{0}$ has a positive contribution from the $W$-exchange box diagram, while both $\Xi_{b b}^{-}$and $\Omega_{b b}^{-}$receive destructive Pauli interference contributions. Hence, it is anticipated that $\tau\left(\Omega_{b b}^{-}\right) \sim \tau\left(\Xi_{b b}^{-}\right)>\tau\left(\Xi_{b b}^{0}\right)$. We are going to show in this study that this is indeed the case. Likewise, a large $W$-exchange contribution to $\Xi_{b c}^{0}$ through the subprocess $c d \rightarrow u s \rightarrow c d$ and a large destructive Pauli interference contribution to $\Xi_{b c}^{+}$(see Fig. 2) will imply a substantial lifetime difference between $\Xi_{b c}^{+}$and $\Xi_{b c}^{0}$, which will be checked in this work.

TABLE I. Lifetimes of doubly charmed baryons in units of $10^{-13} \mathrm{~s}$. The results of [9] are based on the calculation of using $m_{c}=1.73 \pm 0.07 \mathrm{GeV}$ and $m_{s}=0.35 \pm 0.20 \mathrm{GeV}$ from a fit to the LHCb measurement of $\tau\left(\Xi_{c c}^{++}\right)$.

\begin{tabular}{lccc}
\hline \hline & $\Xi_{c c}^{++}$ & $\Xi_{c c}^{+}$ & $\Omega_{c c}^{+}$ \\
\hline Kiselev et al. ('99) [3] & $4.3 \pm 1.1$ & $1.1 \pm 0.3$ & \\
Guberina et al. ('99) [4] & 15.5 & 2.2 & 2.5 \\
Kiselev et al. ('02) [5] & $4.6 \pm 0.5$ & $1.6 \pm 0.5$ & $2.7 \pm 0.6$ \\
Chang et al. ('04) [6] & 6.7 & 2.5 & 2.1 \\
Karliner, Rosner ('14) [7] & 1.85 & 0.53 & \\
Cheng, Shi ('18) [8] & 2.98 & 0.44 & $0.75-1.80$ \\
Berezhnoy et al. ('18) [9] & $2.6 \pm 0.3$ & $1.4 \pm 0.1$ & $1.8 \pm 0.2$ \\
Expt. [2] & $2.56_{-0.26}^{+0.28}$ & & \\
\hline \hline
\end{tabular}


TABLE II. Predicted lifetimes of doubly bottom and charm-bottom baryons in units of $10^{-13} \mathrm{~s}$.

\begin{tabular}{lccccc}
\hline \hline & Likhoded et al. [10] & Kiselev et al. [11] & Kiselev et al. [5] & Karliner et al. [7] & Berezhnoy et al. [9] \\
\hline$\Xi_{b b}^{0}$ & 7.9 & & 7.9 & 3.7 & $5.2 \pm 0.095$ \\
$\Xi_{b b}^{-}$ & 8.0 & & 8.0 & 3.7 & $5.3 \pm 0.096$ \\
$\Omega_{b b}^{-}$ & 8.0 & 8.0 & & $5.3 \pm 0.093$ \\
$\Xi_{b c}^{+}$ & 2.8 & $3.3 \pm 0.8$ & $3.0 \pm 0.4$ & 2.44 & $2.4 \pm 0.2$ \\
$\Xi_{b c}^{0}$ & 2.6 & $2.8 \pm 0.7$ & $2.7 \pm 0.3$ & 0.93 & $2.2 \pm 0.18$ \\
$\Omega_{b c}^{0}$ & 2.1 & & $2.2 \pm 0.4$ & & $1.8 \pm 0.088$ \\
\hline \hline
\end{tabular}

The study of $\mathcal{B}_{b c}$ lifetimes is more complicated than the $\mathcal{B}_{b b}$ case for several reasons. First, besides the spectator effects due to each heavy quark $b$ or $c$, there also exist $W$-exchange and Pauli interference in which both $b$ and $c$ quarks get involved. Second, care must be taken when considering the heavy quark expansion (HQE) for the charm quark. It is known that the HQE in $1 / m_{b}$ works well for bottom hadrons [12]. On the contrary, the HQE to
$1 / m_{c}^{3}$ fails to give a satisfactory description of the lifetimes of both charmed mesons and charmed baryons [12]. Since the charm quark is not heavy, it is thus natural to consider the effects arising from the next order $1 / m_{c}$ expansion. This calls for the subleading $1 / m_{Q}$ corrections to the spectator effects. It turns out that although the relevant dimension-7 spectator effects are in the right direction for explaining the large lifetime ratio of the charmed baryons

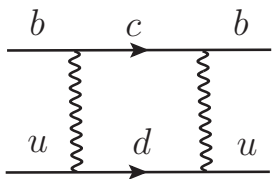

(a)

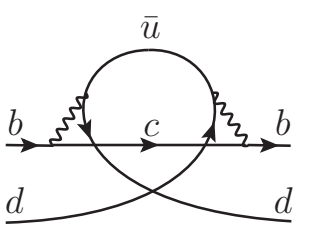

(b)

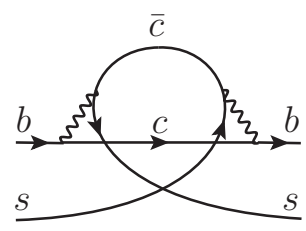

(c)

FIG. 1. Spectator effects in the nonleptonic decays of the doubly bottom baryons: (a) $W$-exchange in $\Xi_{b b}^{0}$ decay and destructive Pauli interference in (b) $\Xi_{b b}^{-}$and (c) $\Omega_{b b}^{-}$decays.
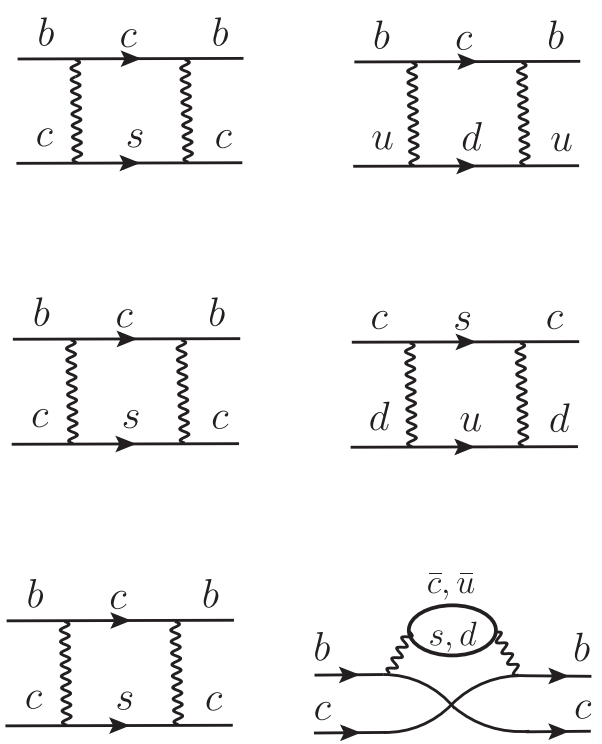

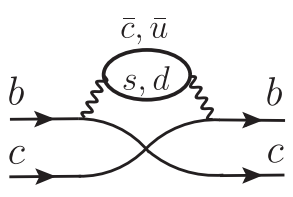

(a)
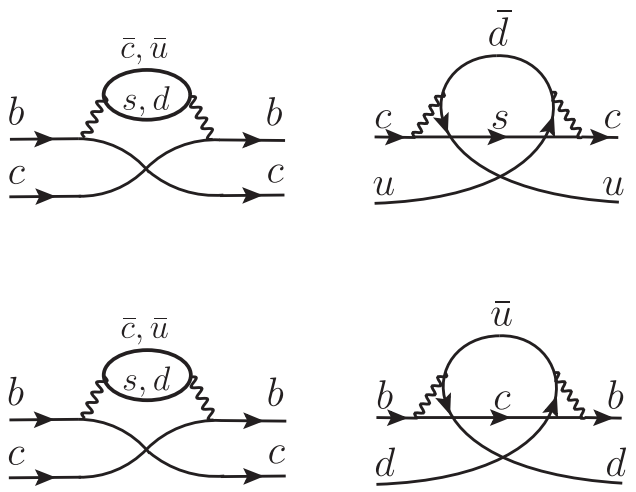

(b)
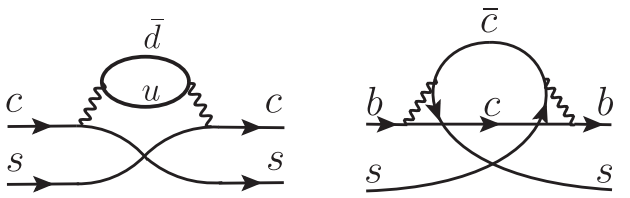

(c)

FIG. 2. Spectator effects in the nonleptonic weak decays of the charm-bottom baryons: (a) $\Xi_{b c}^{+}$, (b) $\Xi_{b c}^{0}$, and (c) $\Omega_{b c}^{0}$. In (a) and (b) there are two $W$-exchange diagrams, one constructive Pauli interference and one destructive Pauli interference. In (c) there are one $W$ exchange, two constructive Pauli interferences and one destructive Pauli interference. 
such as $\tau\left(\Xi_{c}^{+}\right) / \tau\left(\Lambda_{c}^{+}\right)$, the destructive $1 / m_{c}$ corrections to the lifetime of $\Omega_{c}^{0}$ are too large to justify the use of the HQE, namely, the predicted Pauli interference and semileptonic rates for the $\Omega_{c}^{0}$ become negative, which certainly does not make sense. Demanding these rates to be positive for a sensible HQE, it has been conjectured in [12] that the $\Omega_{c}^{0}$ lifetime lies in the range of $(2.3-3.2) \times 10^{-13} \mathrm{~s}$. This is indeed consistent with the new measurement of the $\Omega_{c}^{0}$ lifetime by $\mathrm{LHCb}$ [13] and the new lifetime pattern $\tau\left(\Xi_{c}^{+}\right)>\tau\left(\Omega_{c}^{0}\right)>\tau\left(\Lambda_{c}^{+}\right)>\tau\left(\Xi_{c}^{0}\right)$, contrary to the hierarchy $\tau\left(\Xi_{c}^{+}\right)>\tau\left(\Lambda_{c}^{+}\right)>\tau\left(\Xi_{c}^{0}\right)>\tau\left(\Omega_{c}^{0}\right)$ given in the PDG [14]. This indicates that the $\Omega_{c}^{0}$, which is naively expected to be shortest-lived in the charmed baryon system owing to the large constructive Pauli interference, could live longer than the $\Lambda_{c}^{+}$due to the suppression from next order $1 / m_{c}$ corrections arising from dimension-7 fourquark operators. By the same token, this effect should be also taken into account in both $\mathcal{B}_{b b}$ and $\mathcal{B}_{b c}$ systems.

For the doubly heavy baryons $\mathcal{B}_{b c}$, we shall follow the traditional convention of unprimed states $\Xi_{b c}$ and $\Omega_{b c}$ with the $b c$ diquark being of the axial-vector type $\left(S_{b c}=1\right)$ and primed states $\Xi_{b c}^{\prime}$ and $\Omega_{b c}^{\prime}$ with the scalar type of the heavy diquark $\left(S_{b c}=0\right)$. Theoretical calculations imply that $\mathcal{B}_{b c}^{\prime}$ is heavier than $\mathcal{B}_{b c}{ }^{1}$ Hence, the primed $\mathcal{B}_{b c}^{\prime}$ baryons are dominated by the electromagnetic decay $\mathcal{B}_{b c}^{\prime} \rightarrow \mathcal{B}_{b c} \gamma$.

In this work we shall study the lifetimes of doubly heavy baryons within the framework of the HQE. It is organized as follows. In Sec. II we give the general HQE expressions for inclusive nonleptonic and semileptonic widths. Special attention is paid to the doubly heavy baryon matrix elements of dimension-3 and -5 operators which are somewhat different from the ones of singly heavy baryons. We then proceed to discuss the relevant dimension- 6 and -7 four-quark operators. Evaluation of doubly heavy baryon matrix elements and numerical results are presented in Sec. III. Conclusions are given in Sec. IV.

\section{THEORETICAL FRAMEWORK}

Under the heavy quark expansion, the inclusive nonleptonic decay rate of a doubly heavy baryon $\mathcal{B}_{Q Q^{\prime}}$ containing two heavy quarks $Q Q^{\prime}$ is given by $[17,18]$

$$
\begin{aligned}
\Gamma\left(\mathcal{B}_{Q Q^{\prime}}\right) & =\frac{1}{2 m_{\mathcal{B}_{Q Q^{\prime}}}} \operatorname{Im}\left\langle\mathcal{B}_{Q Q^{\prime}}|T| \mathcal{B}_{Q Q^{\prime}}\right\rangle \\
& =\frac{1}{2 m_{\mathcal{B}_{Q Q^{\prime}}}}\left\langle\mathcal{B}_{Q Q^{\prime}}\left|\int d^{4} x T\left[\mathcal{L}_{W}^{\dagger}(x) \mathcal{L}_{W}(0)\right]\right| \mathcal{B}_{Q Q^{\prime}}\right\rangle,
\end{aligned}
$$

\footnotetext{
${ }^{1}$ Almost all the calculations available in the literature lead to $m_{\Xi_{b c}^{\prime}}>m_{\Xi_{b c}}$ (see, e.g., Fig. 18 of [15]) except the QCD sum-rule calculation of [16] and the lattice QCD calculation of [15]. Note that the definition of primed and unprimed $\mathcal{B}_{b c}$ states in [15] is opposite to the conventional one.
}

in analog to the case of a singly heavy baryon $\mathcal{B}_{Q}$. Through the use of the operator product expansion, the transition operator $T$ can be expressed in terms of local quark operators

$$
\begin{aligned}
\operatorname{Im} T= & \frac{G_{F}^{2} m_{Q}^{5}}{192 \pi^{3}} \xi\left(c_{3, Q} \bar{Q} Q+\frac{c_{5, Q}}{m_{Q}^{2}} \bar{Q} \sigma \cdot G Q+\frac{c_{6, Q}}{m_{Q}^{3}} T_{6}\right. \\
& \left.+\frac{c_{7, Q}}{m_{Q}^{4}} T_{7}+\cdots\right),
\end{aligned}
$$

where $\xi$ is the relevant Cabibbo-Kobayashi-Maskawa (CKM) matrix element, the dimension- $6 T_{6}$ consists of the four-quark operators $(\bar{Q} \Gamma q)(\bar{q} \Gamma Q)$ with $\Gamma$ representing a combination of the Lorentz and color matrices, while a subset of dimension-7 $T_{7}$ is governed by the four-quark operators containing derivative insertions. Hence,

$$
\begin{aligned}
\Gamma\left(\mathcal{B}_{b c}\right)= & \frac{G_{F}^{2} m_{b}^{5}}{192 \pi^{3}} \xi \frac{1}{2 m_{\mathcal{B}_{b c}}}\left\{c_{3, b}\left\langle\mathcal{B}_{b c}|\bar{b} b| \mathcal{B}_{b c}\right\rangle\right. \\
& +\frac{c_{5, b}}{m_{b}^{2}}\left\langle\mathcal{B}_{b c}|\bar{b} \sigma \cdot G b| \mathcal{B}_{b c}\right\rangle+\frac{c_{6, b}}{m_{b}^{3}}\left\langle\mathcal{B}_{b c}\left|T_{6}\right| \mathcal{B}_{b c}\right\rangle \\
& \left.+\frac{c_{7, b}}{m_{b}^{4}}\left\langle\mathcal{B}_{b c}\left|T_{7}\right| \mathcal{B}_{b c}\right\rangle+\cdots\right\} \\
& +\frac{G_{F}^{2} m_{c}^{5}}{192 \pi^{3}} \xi \frac{1}{2 m_{\mathcal{B}_{b c}}}\left\{c_{3, c}\left\langle\mathcal{B}_{b c}|\bar{c} c| \mathcal{B}_{b c}\right\rangle\right. \\
& +\frac{c_{5, c}}{m_{c}^{2}}\left\langle\mathcal{B}_{b c}|\bar{c} \sigma \cdot G c| \mathcal{B}_{b c}\right\rangle+\frac{c_{6, c}}{m_{c}^{3}}\left\langle\mathcal{B}_{b c}\left|T_{6}\right| \mathcal{B}_{b c}\right\rangle \\
& \left.+\frac{c_{7, c}}{m_{c}^{4}}\left\langle\mathcal{B}_{b c}\left|T_{7}\right| \mathcal{B}_{b c}\right\rangle+\cdots\right\} .
\end{aligned}
$$

In the following we shall discuss the contributions from dimension-3, -5, -6 and -7 operators separately.

\section{A. Dimension-3 and -5 operators}

In heavy quark effective theory (HQET), the dimension3 operator $\bar{Q} Q$ in the rest frame has the expression

$\bar{Q} Q=\bar{Q} \gamma_{0} Q-\frac{\bar{Q}(i \vec{D})^{2} Q}{2 m_{Q}^{2}}+\frac{\bar{Q} \sigma \cdot G Q}{4 m_{Q}^{2}}+\mathcal{O}\left(\frac{1}{m_{Q}^{3}}\right)$,

with the normalization

$$
\frac{\left\langle\mathcal{B}_{Q Q^{\prime}}\left|\bar{Q} \gamma_{0} Q\right| \mathcal{B}_{Q Q^{\prime}}\right\rangle}{2 m_{\mathcal{B}_{Q Q^{\prime}}}}=1 .
$$

Hence,

$$
\frac{\left\langle\mathcal{B}_{b c}|\bar{c} c| \mathcal{B}_{b c}\right\rangle}{2 m_{\mathcal{B}_{b c}}}=1-\frac{\mu_{\pi, c}^{2}}{2 m_{c}^{2}}+\frac{\mu_{G, c}^{2}}{2 m_{c}^{2}}+\mathcal{O}\left(\frac{1}{m_{c}^{3}}\right)
$$

where 


$$
\begin{aligned}
\mu_{\pi, c}^{2} & \equiv \frac{1}{2 m_{\mathcal{B}_{b c}}}\left\langle\mathcal{B}_{b c}\left|\bar{c}(i \vec{D})^{2} c\right| \mathcal{B}_{b c}\right\rangle \\
& =-\frac{1}{2 m_{\mathcal{B}_{b c}}}\left\langle\mathcal{B}_{b c}\left|\bar{c}\left(i D_{\perp}\right)^{2} c\right| \mathcal{B}_{b c}\right\rangle=-\lambda_{1}^{c}, \\
\mu_{G, c}^{2} & \equiv \frac{1}{2 m_{\mathcal{B}_{b c}}}\left\langle\mathcal{B}_{b c}\left|\bar{c} \frac{1}{2} \sigma \cdot G c\right| \mathcal{B}_{b c}\right\rangle=d_{H} \lambda_{2}^{c} .
\end{aligned}
$$

The nonperturbative parameters $\lambda_{1}$ and $\lambda_{2}$ are independent of $m_{Q}$ and have the same values for all particles in a given spin-flavor multiplet.

We first consider the nonperturbative parameter $\mu_{\pi}^{2}$. In general, $\mu_{\pi, Q}^{2}=\left\langle p^{2}\right\rangle=\left\langle m_{Q}^{2} v_{Q}^{2}\right\rangle$. The average kinetic energy of the diquark $b c$ and the light quark $q$ is $T=\frac{1}{2} m_{d i} v_{d i}^{2}+\frac{1}{2} m_{q} v_{q}^{2}$, where $m_{d i}\left(m_{q}\right)$ is the mass of the diquark (light quark). This together with the momentum conservation $m_{d i} v_{d i}=m_{q} v_{q}$ leads to

$$
v_{d i}^{2}=\frac{2 m_{q} T}{\left(m_{b}+m_{c}\right)\left(m_{b}+m_{c}+m_{q}\right)} .
$$

As shown in [3], the average kinetic energy $T^{\prime}$ of heavy quarks inside the diquark given by $\frac{1}{2} m_{b}\left(\tilde{v}_{b}^{2}+\tilde{v}_{c}^{2}\right)$ is equal to $T / 2$ due to the color wave function of the diquark. Hence, the average velocity $\tilde{v}_{b}$ of the heavy quark $b$ inside the diquark is $\tilde{v}_{b}^{2}=m_{c} T /\left[m_{b}\left(m_{b}+m_{c}\right)\right]$, where we have applied the momentum conservation $m_{b} \tilde{v}_{b}=m_{c} \tilde{v}_{c}$. As a result, the average velocity $v_{Q}$ of the heavy quark inside the baryon $\mathcal{B}_{b c}$ is [5]

$$
\begin{aligned}
& v_{b}^{2} \approx \tilde{v}_{b}^{2}+v_{d i}^{2}=\frac{m_{c} T}{m_{b}\left(m_{b}+m_{c}\right)}+\frac{2 m_{q} T}{\left(m_{b}+m_{c}\right)\left(m_{b}+m_{c}+m_{q}\right)}, \\
& v_{c}^{2} \approx \tilde{v}_{c}^{2}+v_{d i}^{2}=\frac{m_{b} T}{m_{c}\left(m_{b}+m_{c}\right)}+\frac{2 m_{q} T}{\left(m_{b}+m_{c}\right)\left(m_{b}+m_{c}+m_{q}\right)} .
\end{aligned}
$$

Hence,

$$
\begin{aligned}
& \mu_{\pi, b}^{2}\left(\mathcal{B}_{b c}\right) \simeq m_{b}^{2}\left(\frac{m_{c} T}{m_{b}\left(m_{b}+m_{c}\right)}+\frac{2 m_{q} T}{\left(m_{b}+m_{c}\right)\left(m_{b}+m_{c}+m_{q}\right)}\right), \\
& \mu_{\pi, c}^{2}\left(\mathcal{B}_{b c}\right) \simeq m_{c}^{2}\left(\frac{m_{b} T}{m_{c}\left(m_{b}+m_{c}\right)}+\frac{2 m_{q} T}{\left(m_{b}+m_{c}\right)\left(m_{b}+m_{c}+m_{q}\right)}\right) .
\end{aligned}
$$

We next turn to the parameter $\mu_{G}^{2}$. For the doubly heavy baryon $\mathcal{B}_{Q Q^{\prime}}$, if the heavy diquark acts as a pointlike constitute, its mass is of the form

$$
\begin{aligned}
m_{\mathcal{B}_{b c}}= & m_{b}+m_{c}+\bar{\Lambda}_{\mathcal{B}_{b c}}+\frac{\mu_{\pi, b}^{2}}{2 m_{b}}+\frac{\mu_{\pi, c}^{2}}{2 m_{c}}-\frac{\mu_{G, b}^{2}}{2 m_{b}} \\
& -\frac{\mu_{G, c}^{2}}{2 m_{c}}+\mathcal{O}\left(\frac{1}{m_{Q}^{2}}\right) .
\end{aligned}
$$

There are two distinct chromomagnetic fields inside the $\mathcal{B}_{b c}$ : one is the chromomagnetic field produced by the light quark and the other by the heavy quark. To proceed, let us consider a simple quark model of De Rújula et al. [19]:

$M_{\text {baryon }}=M_{0}+\cdots+\frac{16}{9} \pi \alpha_{s} \sum_{i>j} \frac{\vec{S}_{i} \cdot \vec{S}_{j}}{m_{i} m_{j}}|\psi(0)|^{2}$,

$M_{\text {meson }}=M_{0}+\cdots+\frac{32}{9} \pi \alpha_{s} \frac{\vec{S}_{1} \cdot \vec{S}_{2}}{m_{1} m_{2}}|\psi(0)|^{2}$.

It is well known that the fine structure constant is $-\frac{4}{3} \alpha_{s}$ for $\bar{q} q$ pairs in a meson and $-\frac{2}{3} \alpha_{s}$ for $q q$ pairs in a baryon [19]. This is because the $\bar{q} q$ pair in a meson must be a color singlet, while the $q q$ pair in a baryon is in color antitriplet state. The mass of the doubly heavy baryon $\mathcal{B}_{b c}$ is given by

$$
\begin{aligned}
m_{\mathcal{B}_{b c}}= & m_{b}+m_{c}+\cdots+\frac{16}{9} \pi \alpha_{s}\left[\left(\frac{\vec{S}_{b} \cdot \vec{S}_{q}}{m_{b} m_{q}}+\frac{\vec{S}_{c} \cdot \vec{S}_{q}}{m_{c} m_{q}}\right)\left|\psi^{q, b c}(0)\right|^{2}\right. \\
& \left.+\frac{\vec{S}_{b} \cdot \vec{S}_{c}}{m_{b} m_{c}}\left|\phi^{b c}(0)\right|^{2}\right]
\end{aligned}
$$

where $\psi^{q, b c}(0)$ is the light quark wave function at the origin of the $b c$ diquark and $\phi^{b c}(0)$ is the diquark wave function at the origin.

The matrix elements of spin-spin interactions are given by

$$
\begin{aligned}
\left\langle\mathcal{B}_{b c}\left|\vec{S}_{b} \cdot \vec{S}_{q}\right| \mathcal{B}_{b c}\right\rangle= & \frac{1}{2}\left[S_{b q}\left(S_{b q}+1\right)-S_{b}\left(S_{b}+1\right)\right. \\
& \left.-S_{q}\left(S_{q}+1\right)\right], \\
\left\langle\mathcal{B}_{b c}\left|\vec{S}_{c} \cdot \vec{S}_{q}\right| \mathcal{B}_{b c}\right\rangle= & \frac{1}{2}\left[S_{c q}\left(S_{c q}+1\right)-S_{c}\left(S_{c}+1\right)\right. \\
& \left.-S_{q}\left(S_{q}+1\right)\right],
\end{aligned}
$$

where $S_{b q}\left(S_{c q}\right)$ is the spin of the diquark $b q(c q)$. To evaluate the diquark spins $S_{b q}$ and $S_{c q}$, we need to change the basis from $\left|S, S_{b c}\right\rangle$ to $\left|S, S_{b q}\right\rangle$ or $\left|S, S_{c q}\right\rangle$ with $S$ being the spin of the heavy baryon:

$$
\begin{aligned}
\left|S, S_{b c}\right\rangle= & \sum_{S_{b q}}(-1)^{\left(S+S_{b}+S_{c}+S_{q}\right)} \sqrt{\left(2 S_{b q}+1\right)\left(2 S_{b c}+1\right)} \\
& \times\left\{\begin{array}{ccc}
S_{q} & S_{b} & S_{b q} \\
S_{c} & S & S_{b c}
\end{array}\right\}\left|S, S_{b q}\right\rangle, \\
\left|S, S_{b c}\right\rangle= & \sum_{S_{c q}}(-1)^{\left(S+S_{b}+S_{c}+S_{q}\right)} \sqrt{\left(2 S_{c q}+1\right)\left(2 S_{b c}+1\right)} \\
& \times\left\{\begin{array}{lll}
S_{q} & S_{c} & S_{c q} \\
S_{b} & S & S_{b c}
\end{array}\right\}\left|S, S_{c q}\right\rangle .
\end{aligned}
$$

We find: 


$$
\begin{aligned}
& m_{\Xi_{b c}}=m_{b}+m_{c}+\cdots+\frac{16}{9} \pi \alpha_{s}\left(-\frac{1}{2} \frac{m_{b}+m_{c}}{m_{b} m_{c} m_{q}}\left|\psi^{q, b c}(0)\right|^{2}+\frac{1}{4 m_{b} m_{c}}\left|\phi^{b c}(0)\right|^{2}\right), \\
& m_{\Xi_{b c}^{*}}=m_{b}+m_{c}+\cdots+\frac{16}{9} \pi \alpha_{s}\left(\frac{1}{4} \frac{m_{b}+m_{c}}{m_{b} m_{c} m_{q}}\left|\psi^{q, b c}(0)\right|^{2}+\frac{1}{4 m_{b} m_{c}}\left|\phi^{b c}(0)\right|^{2}\right) .
\end{aligned}
$$

The term proportional to $\left|\psi^{q, b c}(0)\right|^{2}$ can be expressed in terms of the hyperfine mass splitting of $\Xi_{b c}$ :

$$
m_{\Xi_{b c}^{*}}-m_{\Xi_{b c}}=\frac{4}{3} \pi \alpha_{s} \frac{m_{b}+m_{c}}{m_{b} m_{c} m_{q}}\left|\psi^{q, b c}(0)\right|^{2} .
$$

Identifying the last two terms in the parentheses of Eq. (2.16) with $-\mu_{G, b}^{2} /\left(2 m_{b}\right)-\mu_{G, c}^{2} /\left(2 m_{c}\right)$, we obtain

$$
\mu_{G, b(c)}^{2}\left(\Xi_{b c}\right)=\frac{2}{3}\left(m_{\Xi_{b c}^{*}}-m_{\Xi_{b c}}\right) m_{b(c)}-\frac{4}{9} \pi \alpha_{s} \frac{\left|\phi^{b c}(0)\right|^{2}}{m_{c(b)}} .
$$

It has been known that HQET is not the appropriate effective field theory for hadrons with more than one heavy quark. For a singly heavy hadron, the heavy quark kinetic energy is neglected as it occurs as a small $1 / m_{Q}$ correction. For a bound state containing two or more heavy quarks, the heavy quark kinetic energy is very important and cannot be treated as a perturbation. The appropriate theory for dealing such a system is nonrelativistic QCD (NRQCD), in which one has

$$
\bar{Q} g_{s} \sigma \cdot G Q=-2 \psi_{Q}^{\dagger} g_{s} \vec{\sigma} \cdot \vec{B} \psi_{Q}-\frac{1}{m_{Q}} \psi_{Q}^{\dagger} g_{s} \vec{D} \cdot \vec{E} \psi_{Q}+\cdots
$$

in terms of the two-spinor $\psi_{Q}$. According to the counting rule, the Darwin term for the interaction with the chromoelectric field is of the same order of magnitude as the chromomagnetic term [20]. Hence, we get an additional contribution to $\mu_{G}^{2}$ :

$$
\begin{aligned}
& \mu_{G, b}^{2}=\frac{2}{3}\left(m_{\Xi_{b c}^{*}}-m_{\Xi_{b c}}\right) m_{b}-\frac{1}{9} g_{s}^{2} \frac{\left|\phi^{b c}(0)\right|^{2}}{m_{c}}-\frac{1}{6} g_{s}^{2} \frac{\left|\phi^{b c}(0)\right|^{2}}{m_{b}}, \\
& \mu_{G, c}^{2}=\frac{2}{3}\left(m_{\Xi_{b c}^{*}}-m_{\Xi_{b c}}\right) m_{c}-\frac{1}{9} g_{s}^{2} \frac{\left|\phi^{b c}(0)\right|^{2}}{m_{b}}-\frac{1}{6} g_{s}^{2} \frac{\left|\phi^{b c}(0)\right|^{2}}{m_{c}} .
\end{aligned}
$$

The last term in $\mu_{G, b}^{2}$ and $\mu_{G, c}^{2}$ can be obtained by using the equation of motion for the chromoelectric field. It follows that

$$
\frac{\left\langle\Xi_{b c}|\bar{c} c| \Xi_{b c}\right\rangle}{2 m_{\Xi_{b c}}}=1-\frac{1}{2} v_{c}^{2}+\frac{1}{3} \frac{m_{\Xi_{b c}^{*}}-m_{\Xi_{b c}}}{m_{c}}-\frac{1}{18} g_{s}^{2} \frac{\left|\phi^{b c}(0)\right|^{2}}{m_{b} m_{c}^{2}}-\frac{1}{12} g_{s}^{2} \frac{\left|\phi^{b c}(0)\right|^{2}}{m_{c}^{3}} .
$$

This expression is different from the one given in [5]:

$$
\frac{\left\langle\Xi_{b c}|\bar{c} c| \Xi_{b c}\right\rangle}{2 m_{\Xi_{b c}}}=1-\frac{1}{2} v_{c}^{2}+\frac{1}{3} g_{s}^{2} \frac{\left|\phi^{b c}(0)\right|^{2}}{m_{b} m_{c}^{2}}-\frac{1}{6} g_{s}^{2} \frac{\left|\phi^{b c}(0)\right|^{2}}{m_{c}^{3}} .
$$

We notice that the third term in Eq. (2.21) is absent in the above expression. This is because the authors of [5] considered the charm-bottom baryon $\Xi_{b c}^{\prime}$ with the scalar $b c$ diquark so that $S_{b c}=0$. It is straightforward to show that $\left\langle\Xi_{b c}^{\prime}\left|\vec{S}_{b} \cdot \vec{S}_{q}\right| \Xi_{b c}^{\prime}\right\rangle=\left\langle\Xi_{b c}^{\prime}\left|\vec{S}_{c} \cdot \vec{S}_{q}\right| \Xi_{b c}^{\prime}\right\rangle=0$. Hence, the chromomagnetic interaction of the heavy quark with the light quark does not contribute to $\Xi_{b c}^{\prime}$. However, since $\Xi_{b c}^{\prime}$ is heavier than $\Xi_{b c}$, it is dominated by the electromagnetic decay $\Xi_{b c}^{\prime} \rightarrow \Xi_{b c} \gamma$.

The nonleptonic and semileptonic decay rates of the $\mathcal{B}_{b c}$ baryons are then given by

$$
\Gamma^{\operatorname{dec}}\left(\mathcal{B}_{b c}\right)=\frac{G_{F}^{2} m_{c}^{5}}{192 \pi^{3}} \xi\left\{c_{3, c}^{\mathrm{NL}}\left[1-\frac{\mu_{\pi, c}^{2}}{2 m_{c}^{2}}+\frac{\mu_{G, c}^{2}}{2 m_{c}^{2}}\right]+2 c_{5, c}^{\mathrm{NL}} \frac{\mu_{G, c}^{2}}{m_{c}^{2}}\right\}+\frac{G_{F}^{2} m_{b}^{5}}{192 \pi^{3}} \xi\left\{c_{3, b}^{\mathrm{NL}}\left[1-\frac{\mu_{\pi, b}^{2}}{2 m_{b}^{2}}+\frac{\mu_{G, b}^{2}}{2 m_{b}^{2}}\right]+2 c_{5, b}^{\mathrm{NL}} \frac{\mu_{G, b}^{2}}{m_{b}^{2}}\right\}
$$

and

$$
\Gamma^{\mathrm{SL}}\left(\mathcal{B}_{b c}\right)=\frac{G_{F}^{2} m_{c}^{5}}{192 \pi^{3}} \xi\left\{c_{3, c}^{\mathrm{SL}}\left[1-\frac{\mu_{\pi, c}^{2}}{2 m_{c}^{2}}+\frac{\mu_{G, c}^{2}}{2 m_{c}^{2}}\right]+2 c_{5, c}^{\mathrm{SL}} \frac{\mu_{G, c}^{2}}{m_{c}^{2}}\right\}+\frac{G_{F}^{2} m_{b}^{5}}{192 \pi^{3}} \xi\left\{c_{3, b}^{\mathrm{SL}}\left[1-\frac{\mu_{\pi, b}^{2}}{2 m_{b}^{2}}+\frac{\mu_{G, b}^{2}}{2 m_{b}^{2}}\right]+2 c_{5, b}^{\mathrm{SL}} \frac{\mu_{G, b}^{2}}{m_{b}^{2}}\right\},
$$

where the expressions of the coefficients $c_{3, b(c)}$ and $c_{5, b(c)}$ can be found, e.g., in [12]. For doubly bottom baryons $\mathcal{B}_{b b}$, the expressions of $\Gamma^{\mathrm{dec}}\left(\mathcal{B}_{b b}\right)$ and $\Gamma^{\mathrm{SL}}\left(\mathcal{B}_{b b}\right)$ are the same as Eqs. (2.23) and (2.24), respectively, except that the charm quark is replaced by the bottom quark. 


\section{B. Dimension-6 operators}

Defining

$$
\mathcal{T}_{6}=\frac{G_{F}^{2} m_{Q}^{2}}{192 \pi^{3}} \xi c_{6, Q}^{\mathrm{NL}} T_{6}
$$

the dimension-6 four-quark operators in Eq. (2.3) for spectator effects in inclusive decays of doubly charmed baryons $\mathcal{B}_{b c}$ are given by (only Cabibbo-allowed decays being listed here) [11]:

$$
\begin{aligned}
& \mathcal{T}_{6, \text { ann }, b c}^{\mathcal{B}_{b c}}=\frac{G_{F}^{2} m_{b}^{2}}{2 \pi}\left|V_{c b}\right|^{2}\left(1+\sqrt{x_{b}}\right)^{2}\left(1-x_{b+}\right)^{2}\left\{\left(c_{1}^{2}+c_{2}^{2}\right)(\bar{b} b)(\bar{c} c)+2 c_{1} c_{2}(\bar{b} c)(\bar{c} b)\right\}, \\
& \mathcal{T}_{6, \text { ann }, b u}^{\mathcal{B}_{b c}}=\frac{G_{F}^{2} m_{b}^{2}}{2 \pi}\left|V_{c b}\right|^{2}\left(1-x_{b}\right)^{2}\left\{\left(c_{1}^{2}+c_{2}^{2}\right)(\bar{b} b)(\bar{u} u)+2 c_{1} c_{2}(\bar{b} u)(\bar{u} b)\right\}, \\
& \mathcal{T}_{6, \text { ann }, c d}^{\mathcal{B}_{b c}}=\frac{G_{F}^{2} m_{c}^{2}}{2 \pi}\left|V_{c s}\right|^{2}\left(1-x_{c}\right)^{2}\left\{\left(c_{1}^{2}+c_{2}^{2}\right)(\bar{c} c)(\bar{d} d)+2 c_{1} c_{2}(\bar{c} d)(\bar{d} c)\right\} \\
& \mathcal{T}_{6, \text { int }, c u}^{\mathcal{B}_{b c}}=-\frac{G_{F}^{2} m_{c}^{2}}{6 \pi}\left|V_{c s}\right|^{2}\left(1-x_{c}\right)^{2}\left\{c_{1}^{2}\left[\left(1+\frac{1}{2} x_{c}\right)(\bar{c} c)(\bar{u} u)-\left(1+2 x_{c}\right) \bar{c}^{\alpha}\left(1-\gamma_{5}\right) u^{\beta} \bar{u}^{\beta}\left(1+\gamma_{5}\right) c^{\alpha}\right]\right. \\
& \left.+\left(2 c_{1} c_{2}+N_{c} c_{2}^{2}\right)\left[\left(1+\frac{1}{2} x_{c}\right)(\bar{c} u)(\bar{u} c)-\left(1+2 x_{c}\right) \bar{c}\left(1-\gamma_{5}\right) u \bar{u}\left(1+\gamma_{5}\right) c\right]\right\}, \\
& \mathcal{T}_{6, \text { int- }, b d}^{\mathcal{B}_{b c}}=-\frac{G_{F}^{2} m_{b}^{2}}{6 \pi}\left|V_{c b}\right|^{2}\left(1-x_{b}\right)^{2}\left\{c_{1}^{2}\left[\left(1+\frac{1}{2} x_{b}\right)(\bar{b} b)(\bar{d} d)-\left(1+2 x_{b}\right) \bar{b}^{\alpha}\left(1-\gamma_{5}\right) d^{\beta} \bar{d}^{\beta}\left(1+\gamma_{5}\right) b^{\alpha}\right]\right. \\
& \left.+\left(2 c_{1} c_{2}+N_{c} c_{2}^{2}\right)\left[\left(1+\frac{1}{2} x_{b}\right)(\bar{b} d)(\bar{d} b)-\left(1+2 x_{b}\right) \bar{b}\left(1-\gamma_{5}\right) d \bar{d}\left(1+\gamma_{5}\right) b\right]\right\}, \\
& \mathcal{T}_{6, \text { int-,bs }}^{\mathcal{B}_{b c}}=-\frac{G_{F}^{2} m_{b}^{2}}{6 \pi}\left|V_{c b}\right|^{2} \sqrt{1-4 x_{b}}\left\{c_{1}^{2}\left[\left(1-x_{b}\right)(\bar{b} b)(\bar{s} s)-\left(1+2 x_{b}\right) \bar{b}^{\alpha}\left(1-\gamma_{5}\right) s^{\beta} \bar{s}^{\beta}\left(1+\gamma_{5}\right) b^{\alpha}\right]\right. \\
& \left.+\left(2 c_{1} c_{2}+N_{c} c_{2}^{2}\right)\left[\left(1-x_{b}\right)(\bar{b} s)(\bar{s} b)-\left(1+2 x_{b}\right) \bar{b}\left(1-\gamma_{5}\right) s \bar{s}\left(1+\gamma_{5}\right) b\right]\right\}, \\
& \mathcal{T}_{6, \text { int }+, c s}^{\mathcal{B}_{b c}}=-\frac{G_{F}^{2} m_{c}^{2}}{6 \pi}\left|V_{c s}\right|^{2}\left(1-\sqrt{x_{c}}\right)^{2}\left\{c_{2}^{2}\left[(\bar{c} c)(\bar{s} s)-\bar{c}^{\alpha}\left(1-\gamma_{5}\right) s^{\beta} \bar{s}^{\beta}\left(1+\gamma_{5}\right) c^{\alpha}\right]\right. \\
& \left.+\left(2 c_{1} c_{2}+N_{c} c_{1}^{2}\right)\left[(\bar{c} s)(\bar{s} c)-\bar{c}\left(1-\gamma_{5}\right) s \bar{s}\left(1+\gamma_{5}\right) c\right]\right\} \\
& \mathcal{T}_{6, \text { int }+, b c}^{\mathcal{B}_{b c}}=-\frac{G_{F}^{2} m_{b}^{2}}{6 \pi}\left|V_{c b} V_{c s}\right|^{2}\left(1-\sqrt{x_{b}}\right)^{2}\left(1-x_{b-}\right)^{2}\left\{c_{2}^{2}\left[\left(1+\frac{1}{2} x_{b-}\right)(\bar{b} b)(\bar{c} c)-\left(1+2 x_{b-}\right) \bar{b}^{\alpha}\left(1-\gamma_{5}\right) c^{\beta} \bar{c}^{\beta}\left(1+\gamma_{5}\right) b^{\alpha}\right]\right. \\
& \left.+\left(2 c_{1} c_{2}+N_{c} c_{1}^{2}\right)\left[\left(1+\frac{1}{2} x_{b-}\right)(\bar{b} c)(\bar{c} b)-\left(1+2 x_{b-}\right) \bar{b}\left(1-\gamma_{5}\right) c \bar{c}\left(1+\gamma_{5}\right) b\right]\right\} \\
& -\frac{G_{F}^{2} m_{b}^{2}}{6 \pi}\left|V_{c b} V_{u d}\right|^{2}\left(1-\sqrt{x_{b}}\right)^{2}\left\{\cdots \quad \text { with } \quad x_{b-} \rightarrow 0\right\},
\end{aligned}
$$

where $\left(\bar{q}_{1} q_{2}\right) \equiv \bar{q}_{1} \gamma_{\mu}\left(1-\gamma_{5}\right) q_{2}$, and $\alpha, \beta$ are color indices, $x_{c}=m_{s}^{2} / m_{c}^{2}, x_{b}=m_{c}^{2} / m_{b}^{2}$, and $x_{b \pm}=m_{c}^{2} /\left(m_{b} \pm m_{c}\right)^{2}$.

Spectator effects in the weak decays of the doubly charmed baryons $\Xi_{b c}^{+}, \Xi_{b c}^{0}$, and $\Omega_{b c}^{0}$ are depicted in Fig. 2. The first amplitude $\mathcal{T}_{6, a n n, b c}^{\mathcal{B}_{b c}}$ in (2.26) corresponds to the first $W$-exchange diagram in Figs. 2(a)-2(c), which is common to all $\mathcal{B}_{b c}$ baryons. Similarly, the amplitude $\mathcal{T}_{6, a n n, b u}^{\mathcal{B}_{b c}}$ corresponds to the second $W$-exchange diagram in Fig. 2(a). The amplitude $\mathcal{T}_{6, \text { int }+, b c}^{\mathcal{B}_{c c}}$ arises from the constructive Pauli interference of the $c$ quark produced in the $b$ quark decay with the $c$ quark in the wave function of $\mathcal{B}_{b c}$. It corresponds to the third diagram in Figs. 2(a) and 2(b), and the second diagram in Fig. 2(c). The term $\mathcal{T}_{6, \text { int-,cu }}^{\mathcal{B}_{b c}}$ is due to the destructive interference of the $u$ quark, and it occurs in the fourth diagram in Fig. 2(a).

For inclusive semileptonic decays of $\mathcal{B}_{b c}$ baryons, there is a spectator effect originating from the constructive Pauli interference of the $c$ or $s$ quark [21]; that is, the $c(s)$ quark produced in $b \rightarrow c \ell^{-} \bar{\nu}_{\ell}\left(c \rightarrow s \ell^{+} \nu_{\ell}\right)$ has an interference with the $c$ $(s)$ quark in the wave function of $\mathcal{B}_{b c}\left(\Omega_{b c}^{0}\right)$. This amounts to replacing the loop quarks $s \bar{c}$ and $d \bar{u}(u \bar{d})$ in the second (third) diagram of Fig. 2(c) by $\ell^{-} \bar{\nu}_{\ell}\left(\ell^{+} \nu_{\ell}\right)$. It is now ready to deduce this term from $\mathcal{T}_{6, \text { int }, c s}^{\mathcal{B}_{b c}}$ and $\mathcal{T}_{6, \text { int }+ \text {,bc }}^{\mathcal{B}_{b c}}$ in Eq. (2.26) by putting $c_{1}=1, c_{2}=0$, and $N_{c}=1$ : 


$$
\begin{aligned}
& \mathcal{T}_{6, \text { int }, b c}^{\mathcal{B}_{b c} \mathrm{SL}}=-\frac{G_{F}^{2} m_{b}^{2}}{6 \pi}\left|V_{c b}\right|^{2}\left(1-\sqrt{x_{b}}\right)^{2}\left[\left(1+\frac{1}{2} x_{b \ell}\right)(\bar{b} c)(\bar{c} b)-\left(1+2 x_{b \ell}\right) \bar{b}\left(1-\gamma_{5}\right) c \bar{c}\left(1+\gamma_{5}\right) b\right], \\
& \mathcal{T}_{6, \text { int }, c s}^{\mathcal{B}_{b c}, \mathrm{SL}}=-\frac{G_{F}^{2} m_{c}^{2}}{6 \pi}\left|V_{c s}\right|^{2}\left(1-\sqrt{x_{c}}\right)^{2}\left[\left(1+\frac{1}{2} x_{c \ell}\right)(\bar{c} s)(\bar{s} c)-\left(1+2 x_{c \ell}\right) \bar{c}\left(1-\gamma_{5}\right) s \bar{s}\left(1+\gamma_{5}\right) c\right],
\end{aligned}
$$

where $x_{c \ell}=m_{\ell}^{2} / m_{c}^{2}$ and $x_{b \ell}=m_{\ell}^{2} / m_{b}^{2}$.

For doubly bottom baryons $\mathcal{B}_{b b}$, the expressions of $\mathcal{T}_{6, a n n, b u}^{\mathcal{B}_{b b}}, \mathcal{T}_{6, \text { int-,bd }}^{\mathcal{B}_{b b}}$, and $\mathcal{T}_{6, \text { int-,bs }}^{\mathcal{B}_{b b}}$ (see Fig. 1) have the same expressions as $\mathcal{T}_{6, \text { ann,bu }}^{\mathcal{B}_{b c}}, \mathcal{T}_{6, \text { int-,bd }}^{\mathcal{B}_{b c}}$, and $\mathcal{T}_{6, \text { int-,bs }}^{\mathcal{B}_{b c}}$, respectively, in Eq. (2.26). However, there is no additional spectator effect in semileptonic decays of $\mathcal{B}_{b b}$ baryons.

\section{Dimension-7 operators}

To the order of $1 / m_{Q}^{4}$ in the heavy quark expansion in Eq. (2.3), we need to consider dimension-7 operators. For our purposes, we shall focus on the $1 / m_{Q}$ corrections to the spectator effects discussed in the last subsection and neglect the operators with gluon fields. Dimension-7 terms are either the four-quark operators times the spectator quark mass or the four-quark operators with one or two additional derivatives [22,23].

We obtain [12]

$$
\begin{aligned}
\mathcal{T}_{7, a n n, b c}^{\mathcal{B}_{b c}=}= & \frac{G_{F}^{2} m_{b}^{2}}{2 \pi}\left|V_{c b}\right|^{2}\left(1+\sqrt{x_{b}}\right)^{2}\left(1-x_{b+}\right)^{2}\left\{2 c_{1} c_{2}\left[2\left(1+x_{b}\right) P_{3}^{b c}+\left(1-x_{b}\right) P_{5}^{b c}\right]+\left(c_{1}^{2}+c_{2}^{2}\right)\left[2\left(1+x_{b}\right) \tilde{P}_{3}^{b c}+\left(1-x_{b}\right) \tilde{P}_{5}^{b c}\right]\right\}, \\
\mathcal{T}_{7, a n n, b u}^{\mathcal{B}_{b c}}= & \frac{G_{F}^{2} m_{b}^{2}}{2 \pi}\left|V_{c b}\right|^{2}\left(1-x_{b}\right)^{2}\left\{2 c_{1} c_{2}\left[2\left(1+x_{b}\right) P_{3}^{b u}+\left(1-x_{b}\right) P_{5}^{b u}\right]+\left(c_{1}^{2}+c_{2}^{2}\right)\left[2\left(1+x_{b}\right) \tilde{P}_{3}^{b u}+\left(1-x_{b}\right) \tilde{P}_{5}^{b u}\right]\right\}, \\
\mathcal{T}_{7, a n n, c d}^{\mathcal{B}_{b c}}= & \frac{G_{F}^{2} m_{c}^{2}}{2 \pi}\left|V_{c s}\right|^{2}\left(1-x_{c}\right)^{2}\left\{2 c_{1} c_{2}\left[2\left(1+x_{c}\right) P_{3}^{c d}+\left(1-x_{c}\right) P_{5}^{c d}\right]+\left(c_{1}^{2}+c_{2}^{2}\right)\left[2\left(1+x_{c}\right) \tilde{P}_{3}^{c d}+\left(1-x_{c}\right) \tilde{P}_{5}^{c d}\right]\right\}, \\
\mathcal{T}_{7, \text { int,cu }}^{\mathcal{B}_{b c}=}= & \frac{G_{F}^{2} m_{c}^{2}}{6 \pi}\left|V_{c s}\right|^{2}\left(1-x_{c}\right)^{2}\left\{( 2 c _ { 1 } c _ { 2 } + N _ { c } c _ { 2 } ^ { 2 } ) \left[-\left(1-x_{c}\right)\left(1+2 x_{c}\right)\left(P_{1}^{c u}+P_{2}^{c u}\right)+2\left(1+x_{c}+x_{c}^{2}\right) P_{3}^{c u}-12 x_{c}^{2} P_{4}^{c u}\right.\right. \\
& \left.-\left(1-x_{c}\right)\left(1+\frac{1}{2} x_{c}\right) P_{5}^{c u}+\left(1-x_{c}\right)\left(1+2 x_{c}\right) P_{6}^{c u}\right]+c_{1}^{2}\left[-\left(1-x_{c}\right)\left(1+2 x_{c}\right)\left(\tilde{P}_{1}^{c u}+\tilde{P}_{2}^{c u}\right)+2\left(1+x_{c}+x_{c}^{2}\right) \tilde{P}_{3}^{c u}\right. \\
& \left.\left.-12 x_{c}^{2} \tilde{P}_{4}^{c u}-\left(1-x_{c}\right)\left(1+\frac{1}{2} x_{c}\right) \tilde{P}_{5}^{c u}+\left(1-x_{c}\right)\left(1+2 x_{c}\right) \tilde{P}_{6}^{c u}\right]\right\}, \\
\mathcal{T}_{7, \text { int }, b d}^{\mathcal{B}_{b c}}= & \mathcal{T}_{7, \text { int,cu}}^{\mathcal{B}_{b c}}\left(c \rightarrow b, u \rightarrow d, V_{c s} \rightarrow V_{c b}\right), \\
\mathcal{T}_{7, \text { int }, b s}^{\mathcal{B}_{b c}=} & \mathcal{T}_{7, \text { int,bd}}^{\mathcal{B}_{b c}}(d \rightarrow s), \\
\mathcal{T}_{7, \text { int,cs }}^{\mathcal{B}_{b c}}= & \frac{G_{F}^{2} m_{c}^{2}}{6 \pi}\left|V_{c s}\right|^{2}\left(1-\sqrt{x_{c}}\right)^{2}\left\{\left(2 c_{1} c_{2}+N_{c} c_{1}^{2}\right)\left[-P_{1}^{c s}-P_{2}^{c s}+2 P_{3}^{c s}-P_{5}^{c s}+P_{6}^{c s}\right]+c_{2}^{2}\left[-\tilde{P}_{1}^{c s}-\tilde{P}_{2}^{c s}+2 \tilde{P}_{3}^{c s}-\tilde{P}_{5}^{c s}+\tilde{P}_{6}^{c s}\right]\right\}, \\
\mathcal{T}_{7, i n t, b c}^{\mathcal{B}_{b c}}= & \frac{G_{F}^{2} m_{b}^{2}}{6 \pi}\left|V_{c b}\right|^{2}\left(1-\sqrt{x_{b}}\right)^{2}\left\{\left(2 c_{1} c_{2}+N_{c} c_{1}^{2}\right)\left[-P_{1}^{b c}-P_{2}^{b c}+2 P_{3}^{b c}-P_{5}^{b c}+P_{6}^{b c}\right]+c_{2}^{2}\left[-\tilde{P}_{1}^{b c}-\tilde{P}_{2}^{b c}+2 \tilde{P}_{3}^{b c}-\tilde{P}_{5}^{b c}+\tilde{P}_{6}^{b c}\right]\right\},
\end{aligned}
$$

where dimension-7 four-quark operators are defined by $[24]^{2}$

$$
\begin{array}{ll}
P_{1}^{Q q}=\frac{m_{q}}{m_{Q}} \bar{Q}\left(1-\gamma_{5}\right) q \bar{q}\left(1-\gamma_{5}\right) Q, & P_{2}^{Q q}=\frac{m_{q}}{m_{Q}} \bar{Q}\left(1+\gamma_{5}\right) q \bar{q}\left(1+\gamma_{5}\right) Q, \\
P_{3}^{Q q}=\frac{1}{m_{Q}^{2}} \bar{Q} \overleftarrow{D}_{\rho} \gamma_{\mu}\left(1-\gamma_{5}\right) D^{\rho} q \bar{q} \gamma^{\mu}\left(1-\gamma_{5}\right) Q, & P_{4}^{Q q}=\frac{1}{m_{Q}^{2}} \bar{Q} \overleftarrow{D}_{\rho}\left(1-\gamma_{5}\right) D^{\rho} q \bar{q}\left(1+\gamma_{5}\right) Q, \\
\left.P_{5}^{Q q}=\frac{1}{m_{Q}} \bar{Q} \gamma_{\mu}\left(1-\gamma_{5}\right) q \bar{q} \gamma^{\mu}\left(1-\gamma_{5}\right)(i \not)\right) Q, & P_{6}^{Q q}=\frac{1}{m_{Q}} \bar{Q}\left(1-\gamma_{5}\right) q \bar{q}\left(1+\gamma_{5}\right)(i \not D) Q,
\end{array}
$$

\footnotetext{
${ }^{2}$ The $T_{7}$ term in the HQE is suppressed by a factor of $1 / m_{Q}$ relative to $T_{6}$ [see Eq. (2.2)]. However, this suppression factor is absorbed in the definition of $P_{i}^{Q q}$ for later convenience. We shall see below that the hadronic matrix elements of dimension-7 operators are suppressed relative to that of dimension- 6 ones by order $m_{q} / m_{Q}$.
} 
and $\tilde{P}_{i}$ denotes the color-rearranged operator that follows from the expression of $P_{i}$ by interchanging the color indices of the $Q_{i}$ and $q_{j}$ Dirac spinors, e.g., $\tilde{P}_{1}^{Q q}=\frac{m_{q}}{m_{O}} \bar{Q}_{i}\left(1-\gamma_{5}\right) q_{j} \bar{q}_{j}\left(1-\gamma_{5}\right) Q_{i}$.

For doubly bottom baryons $\mathcal{B}_{b b}$, the spectator effects $\mathcal{T}_{7, a n n, b u}^{\mathcal{B}_{b b}}, \mathcal{T}_{7, i n t, b d}^{\mathcal{B}_{b b}}$, and $\mathcal{T}_{7, \text { int bs }}^{\mathcal{B}_{b b}}$ have the same expressions as $\mathcal{T}_{7, a n n, b u}^{\mathcal{B}_{b c}}, \mathcal{T}_{7, \text { int }, b d}^{\mathcal{B}_{b c}}$, and $\mathcal{T}_{7, \text { int,bs }}^{\mathcal{B}_{b c}}$ in Eq. (2.28), respectively.

As for the dimension-7 contributions to semileptonic decays, it can be obtained from $\mathcal{T}_{7 \text {,int }}^{\mathcal{B}_{b c}, b c}$ and $\mathcal{T}_{7 \text {, int }}^{\mathcal{B}_{b c}, c s}$ by setting $c_{1}=1, c_{2}=0$, and $N_{c}=1$. Taking into account the lepton mass corrections, it reads [12]

$$
\begin{aligned}
\mathcal{T}_{7, \text { int }}^{\mathrm{SL}, b c}= & \frac{G_{F}^{2} m_{b}^{2}}{6 \pi}\left|V_{c b}\right|^{2}\left(1-\sqrt{x_{b}}\right)^{2}\left[-\left(1-x_{b \ell}\right)^{2}\right. \\
& \times\left(1+2 x_{b \ell}\right)\left(P_{1}^{b c}+P_{2}^{b c}\right)+2\left(1-x_{b \ell}\right) \\
& \left.\times\left(1+x_{b \ell}+x_{b \ell}^{2}\right) P_{3}^{b c}-12 x_{b \ell}^{2}\left(1-x_{b \ell}\right) P_{4}^{b c}\right], \\
\mathcal{T}_{7, \text { int }}^{\mathrm{SL}, c s}= & \frac{G_{F}^{2} m_{c}^{2}}{6 \pi}\left|V_{c s}\right|^{2}\left(1-\sqrt{x_{c}}\right)^{2}\left[-\left(1-x_{c \ell}\right)^{2}\right. \\
& \times\left(1+2 x_{c \ell}\right)\left(P_{1}^{c s}+P_{2}^{c s}\right)+2\left(1-x_{c \ell}\right) \\
& \left.\times\left(1+x_{c \ell}+x_{c \ell}^{2}\right) P_{3}^{c s}-12 x_{c \ell}^{2}\left(1-x_{c \ell}\right) P_{4}^{c s}\right],
\end{aligned}
$$

where $x_{Q \ell}=\left(m_{\ell} / m_{Q}\right)^{2}$.

\section{LIFETIMES OF DOUBLY HEAVY BARYONS $\mathcal{B}_{b c}$ AND $\mathcal{B}_{b b}$}

\section{A. Baryon matrix elements}

The spectator effects in inclusive decays of the charm-bottom baryons $\mathcal{B}_{b c}$ arising from dimension-6 and dimension-7 operators are given by Eqs. (2.26)-(2.28) and Eq. (2.30), respectively. We shall rely on the quark model to evaluate the baryon matrix elements of fourquark operators. Since the heavy $b c$ diquark of $\mathcal{B}_{b c}$ is of the axial-vector type, its flavor-spin wave function is given by

$$
\begin{aligned}
\mathcal{B}_{b c}= & \frac{1}{6}\left(2 b^{\uparrow} c^{\uparrow} q^{\downarrow}-b^{\uparrow} c^{\downarrow} q^{\uparrow}-b^{\downarrow} c^{\uparrow} q^{\uparrow}+2 c^{\uparrow} b^{\uparrow} q^{\downarrow}\right. \\
& \left.-c^{\uparrow} b^{\downarrow} q^{\uparrow}-c^{\downarrow} b^{\uparrow} q^{\uparrow}+(13)+(23)\right) .
\end{aligned}
$$

In the nonrelativistic quark model we have (see Appendix B of [12] for the detail)

$$
\left\langle\Xi_{b c}|(\bar{c} c)(\bar{q} q)| \Xi_{b c}\right\rangle=6 m_{\Xi_{b c}}\left|\psi^{q, b c}(0)\right|^{2},
$$

where we have taken into account the normalization of the matrix element $\left\langle\mathcal{B}_{Q Q^{\prime}}|Q \not \phi Q| \mathcal{B}_{Q Q^{\prime}}\right\rangle$ or $\left\langle\mathcal{B}_{Q Q^{\prime}}\left|Q^{\prime} \not Q^{\prime}\right| \mathcal{B}_{Q Q^{\prime}}\right\rangle$ to $2 m_{B_{Q Q^{\prime}}}$. The relevant $\mathcal{B}_{b c}$ baryon matrix elements of dimension-6 operators are

$$
\begin{aligned}
\left\langle\mathcal{B}_{b c}|(\bar{Q} q)(\bar{q} Q)| \mathcal{B}_{b c}\right\rangle & =-6 m_{\mathcal{B}_{b c}}\left|\psi^{q, b c}(0)\right|^{2}, \\
\left\langle\mathcal{B}_{b c}|(\bar{Q} Q)(\bar{q} q)| \mathcal{B}_{b c}\right\rangle & =6 m_{\mathcal{B}_{b c}}\left|\psi^{q, b c}(0)\right|^{2} \tilde{B}, \\
\left\langle\mathcal{B}_{b c}\left|\bar{Q}\left(1-\gamma_{5}\right) q \bar{q}\left(1+\gamma_{5}\right) Q\right| \mathcal{B}_{b c}\right\rangle & =-m_{\mathcal{B}_{b c}}\left|\psi^{q, b c}(0)\right|^{2}, \\
\left\langle\mathcal{B}_{b c}\left|\bar{Q}^{\alpha}\left(1-\gamma_{5}\right) q^{\beta} \bar{q}^{\beta}\left(1+\gamma_{5}\right) Q^{\alpha}\right| \mathcal{B}_{b c}\right\rangle & =m_{\mathcal{B}_{b c}}\left|\psi^{q, b c}(0)\right|^{2} \tilde{B},
\end{aligned}
$$

with $Q=b, c$. The parameter $\tilde{B}$ is defined by

$$
\left\langle\mathcal{B}_{b c}|(\bar{Q} Q)(\bar{q} q)| \mathcal{B}_{b c}\right\rangle=-\tilde{B}\left\langle\mathcal{B}_{b c}|(\bar{Q} q)(\bar{q} Q)| \mathcal{B}_{b c}\right\rangle .
$$

Since the color wave function for a baryon is totally antisymmetric, the matrix element of $(\bar{Q} Q)(\bar{q} q)$ is the same as that of $(\bar{Q} q)(\bar{q} Q)$ except for a sign difference. That is, $\tilde{B}=1$ under the valence-quark approximation.

For the $\mathcal{B}_{b c}$ matrix elements of four-quark operators involved with both $b$ and $c$ quarks, we obtain

$$
\begin{aligned}
\left\langle\mathcal{B}_{b c}|(\bar{b} c)(\bar{c} b)| \mathcal{B}_{b c}\right\rangle & =0, \\
\left\langle\mathcal{B}_{b c}|(\bar{b} b)(\bar{c} c)| \mathcal{B}_{b c}\right\rangle & =0, \\
\left\langle\mathcal{B}_{b c}\left|\bar{b}\left(1-\gamma_{5}\right) c \bar{c}\left(1+\gamma_{5}\right) b\right| \mathcal{B}_{b c}\right\rangle & =2 m_{\mathcal{B}_{b c}}\left|\phi^{b c}(0)\right|^{2}, \\
\left\langle\mathcal{B}_{b c}\left|\bar{b}^{\alpha}\left(1-\gamma_{5}\right) c^{\beta} \bar{c}^{\beta}\left(1+\gamma_{5}\right) b^{\alpha}\right| \mathcal{B}_{b c}\right\rangle & =-2 m_{\mathcal{B}_{b c}}\left|\phi^{b c}(0)\right|^{2} \tilde{B}
\end{aligned}
$$

It should be remarked that the $\mathcal{B}_{b c}$ matrix elements of the four-quark operators $(\bar{b} c)(\bar{c} b)$ and $(\bar{b} b)(\bar{c} c)$ vanish in the nonrelativistic quark model but not in the MIT bag model. However, for the reason of consistency, we will stick to the former model. Note that our expressions of the $\mathcal{B}_{b c}$ matrix elements of dimension- 6 operators Eqs. (3.3) and (3.5) are different from that given in $[5,11]$, in which the spin of the $b c$ diquark is treated to be zero.

Likewise, the $\mathcal{B}_{b c}$ matrix elements of the dimension-7 operators $P_{i}^{Q q}$ read 


$$
\begin{aligned}
\left\langle\mathcal{B}_{b c}\left|P_{1}^{Q q}\right| \mathcal{B}_{b c}\right\rangle & =\left\langle\mathcal{B}_{b c}\left|P_{2}^{Q q}\right| \mathcal{B}_{b c}\right\rangle \\
& =\frac{3}{2} m_{\mathcal{B}_{b c}}\left|\psi^{q, b c}(0)\right|^{2}\left(\frac{m_{\mathcal{B}_{b c}}^{2}-m_{\{b c\}}^{2}}{m_{Q}\left(m_{b}+m_{c}\right)}\right) \eta_{1,2}^{q}, \\
\left\langle\mathcal{B}_{b c}\left|P_{3}^{Q q}\right| \mathcal{B}_{b c}\right\rangle & =3\left\langle\mathcal{B}_{b c}\left|P_{4}^{Q q}\right| \mathcal{B}_{b c}\right\rangle \\
& =-3 m_{\mathcal{B}_{b c}}\left|\psi^{q, b c}(0)\right|^{2}\left(\frac{m_{\mathcal{B}_{b c}}^{2}-m_{\{b c\}}^{2}}{m_{Q}\left(m_{b}+m_{c}\right)}\right) \eta_{3,4}^{q},
\end{aligned}
$$

where the parameters $\eta_{i}^{q}$ are expected to be of order unity, and $m_{\{b c\}}$ is the mass of the axial-vector $b c$ diquark. In the derivation of Eq. (3.6) we have applied the relations $p_{b} \approx m_{b} v$ and $p_{c} \approx m_{c} v$ as the $b c$ system has been treated as a diquark. It is then straightforward to show that

$$
\begin{aligned}
\frac{p_{b} \cdot p_{q}}{m_{b}^{2}} & \approx \frac{1}{2} \frac{m_{\mathcal{B}_{b c}}^{2}-m_{\{b c\}}^{2}}{m_{b}\left(m_{b}+m_{c}\right)}, \\
\frac{p_{c} \cdot p_{q}}{m_{c}^{2}} & \approx \frac{1}{2} \frac{m_{\mathcal{B}_{b c}}^{2}-m_{\{b c\}}^{2}}{m_{c}\left(m_{b}+m_{c}\right)} .
\end{aligned}
$$

Therefore, the matrix elements of dimension-7 operators are suppressed by a factor of $m_{q} / m_{Q}$ relative to that of dimension- 6 ones. Matrix elements of the dimension- 7 operators $P_{i}^{b c}$ read

$$
\begin{aligned}
\left\langle\mathcal{B}_{b c}\left|P_{1}^{b c}\right| \mathcal{B}_{b c}\right\rangle & =\left\langle\mathcal{B}_{b c}\left|P_{2}^{b c}\right| \mathcal{B}_{b c}\right\rangle \\
& =2 m_{\mathcal{B}_{b c}}\left(\frac{m_{c}}{m_{b}}\right)\left|\phi^{b c}(0)\right|^{2} \eta_{1,2}^{q}, \\
\left\langle\mathcal{B}_{b c}\left|P_{3}^{b c}\right| \mathcal{B}_{b c}\right\rangle & =0, \\
\left\langle\mathcal{B}_{b c}\left|P_{4}^{b c}\right| \mathcal{B}_{b c}\right\rangle & =-4 m_{\mathcal{B}_{b c}}\left(\frac{m_{c}}{m_{b}}\right)\left|\phi^{b c}(0)\right|^{2} \eta_{4}^{q} .
\end{aligned}
$$

They are suppressed by a factor of $m_{c} / m_{b}$ relative to the matrix elements of dimension- 6 operators. For the matrix elements of the operators $\tilde{P}_{i}^{Q q}$, we introduce a parameter $\tilde{\beta}_{i}^{q}$ in analog to Eq. (3.4):

$$
\left\langle\mathcal{B}_{b c}\left|\tilde{P}_{i}^{Q q}\right| \mathcal{B}_{b c}\right\rangle=-\tilde{\beta}_{i}^{q}\left\langle\mathcal{B}_{b c}\left|P_{i}^{Q q}\right| \mathcal{B}_{b c}\right\rangle
$$

so that $\tilde{\beta}_{i}^{q}=1$ under the valence quark approximation.

The flavor-spin wave function of $\mathcal{B}_{b b}$ is given by

$$
\mathcal{B}_{b b}=\frac{1}{\sqrt{18}}\left(2 b^{\uparrow} b^{\uparrow} q^{\downarrow}-b^{\uparrow} b^{\downarrow} q^{\uparrow}-b^{\downarrow} b^{\uparrow} q^{\uparrow}+(13)+(23)\right) .
$$

Then the relevant $\mathcal{B}_{b b}$ matrix elements have the expressions

$$
\begin{aligned}
\left\langle\mathcal{B}_{b b}|(\bar{b} q)(\bar{q} b)| \mathcal{B}_{b b}\right\rangle & =-12 m_{\mathcal{B}_{b b}}\left|\psi^{q, b b}(0)\right|^{2}, \\
\left\langle\mathcal{B}_{b b}|(\bar{b} b)(\bar{q} q)| \mathcal{B}_{b b}\right\rangle & =12 m_{\mathcal{B}_{b b}}\left|\psi^{q, b b}(0)\right|^{2} \tilde{B}, \\
\left\langle\mathcal{B}_{b b}\left|\bar{b}\left(1-\gamma_{5}\right) q \bar{q}\left(1+\gamma_{5}\right) b\right| \mathcal{B}_{b b}\right\rangle & =-2 m_{\mathcal{B}_{b b}}\left|\psi^{q, b b}(0)\right|^{2}, \\
\left\langle\mathcal{B}_{b b}\left|\bar{b}^{\alpha}\left(1-\gamma_{5}\right) q^{\beta} \bar{q}^{\beta}\left(1+\gamma_{5}\right) b^{\alpha}\right| \mathcal{B}_{b b}\right\rangle & =2 m_{\mathcal{B}_{b b}}\left|\psi^{q, b b}(0)\right|^{2} \tilde{B},
\end{aligned}
$$

$$
\begin{aligned}
& \left\langle\mathcal{B}_{b b}\left|P_{1}^{b q}\right| \mathcal{B}_{b b}\right\rangle=\left\langle\mathcal{B}_{b b}\left|P_{2}^{b q}\right| \mathcal{B}_{b b}\right\rangle=\frac{3}{2} m_{\mathcal{B}_{b b}}\left|\psi^{q, b b}(0)\right|^{2}\left(\frac{m_{\mathcal{B}_{b b}}^{2}-m_{\{b b\}}^{2}}{m_{b}^{2}}\right) \eta_{1,2}^{q}, \\
& \left\langle\mathcal{B}_{b b}\left|P_{3}^{b q}\right| \mathcal{B}_{b b}\right\rangle=6\left\langle\mathcal{B}_{b b}\left|P_{4}^{b q}\right| \mathcal{B}_{b b}\right\rangle=-3 m_{\mathcal{B}_{b b}}\left|\psi^{q, b b}(0)\right|^{2}\left(\frac{m_{\mathcal{B}_{b b}}^{2}-m_{\{b b\}}^{2}}{m_{b}^{2}}\right) \eta_{3,4}^{q} .
\end{aligned}
$$

In numerical calculations, we shall take $m_{\{b c\}}$ to be $6526 \mathrm{MeV}$ and $m_{\{b b\}} 9978 \mathrm{MeV}$ obtained from the relativistic quark model [25].

We are ready to evaluate the spectator effects in $\mathcal{B}_{b c}$ decays given by

$$
\Gamma^{\mathrm{spec}}\left(\mathcal{B}_{b c}\right)=\frac{\left\langle\mathcal{B}_{b c}\left|\mathcal{T}_{6}+\mathcal{T}_{7}\right| \mathcal{B}_{b c}\right\rangle}{2 m_{\mathcal{B}_{b c}}}
$$


The results are

$$
\begin{aligned}
& \Gamma_{\text {ann }, b c}^{\mathcal{B}_{b c}}=-\frac{G_{F}^{2} m_{b}^{2}}{2 \pi}\left|V_{c b}\right|^{2}\left(1+\sqrt{x_{b}}\right)^{2}\left(1+x_{b}\right)\left(1-x_{b+}\right)^{2}\left(\tilde{\beta}\left(c_{1}^{2}+c_{2}^{2}\right)-2 c_{1} c_{2}\right) \eta\left(\frac{m_{c}}{m_{b}}\right)\left|\phi^{b c}(0)\right|^{2}, \\
& \Gamma_{\text {ann }, b u}^{\mathcal{B}_{b c}}=\frac{3 G_{F}^{2} m_{b}^{2}}{\pi}\left|V_{c b}\right|^{2}\left(1-x_{b}\right)^{2}\left|\psi^{q, b c}(0)\right|^{2}\left\{\left(\tilde{B}\left(c_{1}^{2}+c_{2}^{2}\right)-2 c_{1} c_{2}\right)+\left(1+x_{b}\right)\left(\tilde{\beta}\left(c_{1}^{2}+c_{2}^{2}\right)-2 c_{1} c_{2}\right) \eta\left(\frac{m_{\mathcal{B}_{b c}}^{2}-m_{\{b c\}}^{2}}{m_{b}\left(m_{b}+m_{c}\right)}\right)\right\}, \\
& \Gamma_{\text {ann }, c d}^{\mathcal{B}_{b c}}=\frac{3}{2} \frac{G_{F}^{2} m_{c}^{2}}{\pi}\left|V_{c s}\right|^{2}\left(1-x_{c}\right)^{2}\left|\psi^{q, b c}(0)\right|^{2}\left\{\left(\tilde{B}\left(c_{1}^{2}+c_{2}^{2}\right)-2 c_{1} c_{2}\right)+\left(1+x_{c}\right)\left(\tilde{\beta}\left(c_{1}^{2}+c_{2}^{2}\right)-2 c_{1} c_{2}\right) \eta\left(\frac{m_{\mathcal{B}_{b c}}^{2}-m_{\{b c\}}^{2}}{m_{c}\left(m_{b}+m_{c}\right)}\right)\right\} \text {, } \\
& \Gamma_{\text {int }_{-}, c u}^{\mathcal{B}_{b c}}=-\frac{G_{F}^{2} m_{c}^{2}}{12 \pi}\left|V_{c s}\right|^{2}\left(1-x_{c}\right)^{2}\left|\psi^{q, b c}(0)\right|^{2}\left\{\left(\tilde{B} c_{1}^{2}-2 c_{1} c_{2}-N_{c} c_{2}^{2}\right)\left(5+x_{c}\right)\right. \\
& \left.-9\left(\tilde{\beta} c_{1}^{2}-2 c_{1} c_{2}-N_{c} c_{2}^{2}\right)\left(1+x_{c}-\frac{2}{3} x_{c}^{2}\right) \eta\left(\frac{m_{\mathcal{B}_{b c}}^{2}-m_{\{b c\}}^{2}}{m_{c}\left(m_{b}+m_{c}\right)}\right)\right\}, \\
& \Gamma_{\text {int_ }, b d}^{\mathcal{B}_{b c}}=-\frac{G_{F}^{2} m_{b}^{2}}{12 \pi}\left|V_{c b}\right|^{2}\left(1-x_{b}\right)^{2}\left|\psi^{q, b c}(0)\right|^{2}\left\{\left(\tilde{B} c_{1}^{2}-2 c_{1} c_{2}-N_{c} c_{2}^{2}\right)\left(5+x_{b}\right)\right. \\
& \left.-9\left(\tilde{\beta} c_{1}^{2}-2 c_{1} c_{2}-N_{c} c_{2}^{2}\right)\left(1+x_{b}-\frac{2}{3} x_{b}^{2}\right) \eta\left(\frac{m_{\mathcal{B}_{b c}}^{2}-m_{\{b c\}}^{2}}{m_{b}\left(m_{b}+m_{c}\right)}\right)\right\}, \\
& \Gamma_{\text {int }, b s}^{\mathcal{B}_{b c}}=-\frac{G_{F}^{2} m_{b}^{2}}{12 \pi}\left|V_{c b}\right|^{2} \sqrt{1-4 x_{b}}\left|\psi^{s, b c}(0)\right|^{2}\left\{\left(\tilde{B} c_{1}^{2}-2 c_{1} c_{2}-N_{c} c_{2}^{2}\right)\left(5+x_{b}\right)\right. \\
& \left.-9\left(\tilde{\beta} c_{1}^{2}-2 c_{1} c_{2}-N_{c} c_{2}^{2}\right)\left(1+x_{b}-\frac{2}{3} x_{b}^{2}\right) \eta\left(\frac{m_{\mathcal{B}_{b c}}^{2}-m_{\{b c\}}^{2}}{m_{b}\left(m_{b}+m_{c}\right)}\right)\right\}, \\
& \Gamma_{\mathrm{int}_{+}, c s}^{\mathcal{B}_{b c}}=\frac{G_{F}^{2} m_{c}^{2}}{12 \pi}\left|V_{c s}\right|^{2}\left(1-\sqrt{x_{c}}\right)^{2}\left|\psi^{s, b c}(0)\right|^{2}\left\{5\left(2 c_{1} c_{2}+N_{c} c_{1}^{2}-\tilde{B} c_{2}^{2}\right)-9\left(2 c_{1} c_{2}+N_{c} c_{1}^{2}-\tilde{\beta} c_{2}^{2}\right) \eta\left(\frac{m_{\mathcal{B}_{b c}}^{2}-m_{\{b c\}}^{2}}{m_{c}\left(m_{b}+m_{c}\right)}\right)\right\}, \\
& \Gamma_{\mathrm{int}_{+}, b c}^{\mathcal{B}_{b c}}=\frac{G_{F}^{2} m_{b}^{2}}{6 \pi}\left|V_{c b} V_{c s}\right|^{2}\left(1-\sqrt{x_{b}}\right)^{2}\left|\phi^{b c}(0)\right|^{2}\left\{\left(2 c_{1} c_{2}+N_{c} c_{1}^{2}-\tilde{B} c_{2}^{2}\right)\left(1-x_{b-}\right)^{2}\left(1+2 x_{b-}\right)-2\left(2 c_{1} c_{2}+N_{c} c_{1}^{2}-\tilde{\beta} c_{2}^{2}\right) \eta\left(\frac{m_{c}}{m_{b}}\right)\right\} \\
& +\frac{G_{F}^{2} m_{b}^{2}}{6 \pi}\left|V_{c b} V_{u d}\right|^{2}\left(1-\sqrt{x_{b}}\right)^{2}\left|\phi^{b c}(0)\right|^{2}\left\{\cdots \text { with } x_{b-} \rightarrow 0\right\},
\end{aligned}
$$

and

$$
\begin{aligned}
& \Gamma_{\mathrm{int}}^{\mathrm{SL}, b c}=\frac{G_{F}^{2} m_{b}^{2}}{6 \pi}\left|V_{c b}\right|^{2}\left(1-\sqrt{x_{b}}\right)^{2}\left|\phi^{b c}(0)\right|^{2}\left[1-2\left(\frac{m_{c}}{m_{b}}\right)\right], \\
& \Gamma_{\mathrm{int}}^{\mathrm{SL}, c s}=\frac{G_{F}^{2} m_{c}^{2}}{12 \pi}\left|V_{c s}\right|^{2}\left(1-\sqrt{x_{c}}\right)^{2}\left|\psi^{s, b c}(0)\right|^{2}\left[5-9\left(\frac{m_{\mathcal{B}_{b c}}^{2}-m_{\{b c\}}^{2}}{m_{c}\left(m_{b}+m_{c}\right)}\right)\right] .
\end{aligned}
$$

Except for the weak annihilation term, the expression of Pauli interference will be very lengthy if the hadronic parameters $\eta_{i}^{q}$ and $\tilde{\beta}_{i}^{q}$ are all treated to be different from each other. Since in realistic calculations we will set $\tilde{\beta}_{i}^{q}\left(\mu_{h}\right)=1$ under the valence quark approximation and put $\eta_{i}^{q}$ to unity, we shall assume for simplicity that $\eta_{i}^{q}=\eta$ and $\tilde{\beta}_{i}^{q}=\tilde{\beta}$.

For the Wilson coefficients in Eqs. (3.14) and (3.15), we choose the scale $\mu$ to be $m_{b}\left(m_{c}\right)$ for the $b(c)$ quark decay and for the spectator effect involved the $b$ (c) quark. For example, we choose $\mu \approx m_{b}$ for the Wilson coefficients in $\Gamma_{\text {int }_{b}, b d}^{\mathcal{B}_{b c}}$ and $\mu \approx m_{c}$ for the Wilson coefficients in $\Gamma_{\text {int }_{+}, c s}^{\mathcal{B}_{b c}}$. For the spectator effect involved both $b$ and $c$ quarks, e.g., $\Gamma_{\text {int }_{+}, b c}^{\mathcal{B}_{b c}}$, we shall follow [20] to set $\mu=2 m_{r}$ with $m_{r}$ being the reduced mass $m_{b} m_{c} /\left(m_{b}+m_{c}\right)$.

There are two quantities in Eqs. (3.14) and (3.15) which we need to know, namely $\left|\psi^{q, b c}(0)\right|$ and $\left|\phi^{b c}(0)\right|$. From Eq. (2.17) we see that the wave function of the light quark at the origin of the $b c$ diquark $\left|\psi^{q, b c}(0)\right|$ is related to the hyperfine mass splitting of $\Xi_{b c}$. To remove the dependence on the light quark mass $m_{q}$, we notice that the hyperfine mass splitting of $B$ mesons is given by

$$
m_{B^{*}}-m_{B}=\frac{32}{9} \alpha_{s} \pi \frac{\left|\psi_{B}^{b \bar{q}}(0)\right|^{2}}{m_{b} m_{q}} .
$$


Hence,

$$
\begin{aligned}
\left|\psi^{q, b c}(0)\right|^{2} & =\frac{8}{3} \frac{\alpha_{s}\left(m_{b}\right)}{\alpha_{s}\left(2 m_{r}\right)} \frac{m_{c}}{m_{b}+m_{c}} \frac{m_{\Xi_{b c}^{*}}-m_{\Xi_{b c}}}{m_{B^{*}}-m_{B}}\left|\psi_{B}^{b \bar{q}}(0)\right|^{2} \\
& \equiv r_{\Xi_{b c}}\left|\psi_{B}^{b \bar{q}}(0)\right|^{2} \\
\left|\psi^{s, b c}(0)\right|^{2} & =\frac{8}{3} \frac{\alpha_{s}\left(m_{b}\right)}{\alpha_{s}\left(2 m_{r}\right)} \frac{m_{c}}{m_{b}+m_{c}} \frac{m_{\Omega_{b c}^{*}}-m_{\Omega_{b c}}}{m_{B_{s}^{*}}-m_{B_{s}}}\left|\psi_{B_{s}}^{b \bar{s}}(0)\right|^{2} \\
& \equiv r_{\Omega_{b c}}\left|\psi_{B_{s}}^{b \bar{s}}(0)\right|^{2}
\end{aligned}
$$

where the $B$ meson wave functions at the origin squared are given by

$$
\left|\psi_{B}^{b \bar{q}}(0)\right|^{2}=\frac{1}{12} f_{B}^{2} m_{B}, \quad\left|\psi_{B_{s}}^{b \bar{s}}(0)\right|^{2}=\frac{1}{12} f_{B_{s}}^{2} m_{B_{s}} .
$$

As for the wave function of the diquark $Q Q^{\prime}$ at the origin, we shall use [26]

$$
\begin{aligned}
\left|\phi^{c c}(0)\right|^{2} & =0.039 \mathrm{GeV}^{3}, \\
\left|\phi^{b c}(0)\right|^{2} & =0.065 \mathrm{GeV}^{3}, \quad\left|\phi^{b b}(0)\right|^{2}=0.152 \mathrm{GeV}^{3} .
\end{aligned}
$$

For the doubly bottom $\mathcal{B}_{b b}$ baryons, we have

$$
\begin{aligned}
\Gamma_{\text {ann, } b u}^{\Xi_{b b}^{0}}= & 3 \frac{G_{F}^{2} m_{b}^{2}}{\pi}\left|V_{c b}\right|^{2}\left(1-x_{b}\right)^{2}\left|\psi^{q, b b}(0)\right|^{2}\left\{\left(\tilde{B}\left(c_{1}^{2}+c_{2}^{2}\right)-2 c_{1} c_{2}\right)+\frac{1}{2}\left(1+x_{b}\right)\left(\tilde{\beta}\left(c_{1}^{2}+c_{2}^{2}\right)-2 c_{1} c_{2}\right) \eta\left(\frac{m_{\Xi_{b b}}^{2}-m_{\{b b\}}^{2}}{m_{b}^{2}}\right)\right\} \\
\Gamma_{\text {int }, b d}^{\Xi_{b b}^{-}}= & -\frac{G_{F}^{2} m_{b}^{2}}{6 \pi}\left|V_{c b}\right|^{2}\left(1-x_{b}\right)^{2}\left|\psi^{q, b b}(0)\right|^{2}\left\{\left(\tilde{B} c_{1}^{2}-2 c_{1} c_{2}-N_{c} c_{2}^{2}\right)\left(5+x_{b}\right)\right. \\
& \left.-\frac{9}{2}\left(\tilde{\beta} c_{1}^{2}-2 c_{1} c_{2}-N_{c} c_{2}^{2}\right)\left(1+x_{b}-\frac{2}{3} x_{b}^{2}\right) \eta\left(\frac{m_{\Xi_{b b}}^{2}-m_{\{b b\}}^{2}}{m_{b}^{2}}\right)\right\} \\
\Gamma_{\text {int }_{-}, b s}^{\Omega_{b b}^{-}}= & -\frac{G_{F}^{2} m_{b}^{2}}{6 \pi}\left|V_{c b}\right|^{2}\left(1-x_{b}\right)^{2}\left|\psi^{s, b b}(0)\right|^{2}\left\{\left(\tilde{B} c_{1}^{2}-2 c_{1} c_{2}-N_{c} c_{2}^{2}\right)\left(5+x_{b}\right)\right. \\
& \left.-\frac{9}{2}\left(\tilde{\beta} c_{1}^{2}-2 c_{1} c_{2}-N_{c} c_{2}^{2}\right)\left(1+x_{b}-\frac{2}{3} x_{b}^{2}\right) \eta\left(\frac{m_{\Omega_{b b}}^{2}-m_{\{b b\}}^{2}}{m_{b}^{2}}\right)\right\}
\end{aligned}
$$

where the wave function of the light quark at the origin of the $b b$ diquark $\left|\psi^{q, b b}(0)\right|$ is given by

$$
\begin{aligned}
\left|\psi^{q, b b}(0)\right|^{2} & =\frac{4}{3} \frac{m_{\Xi_{b b}^{*}}-m_{\Xi_{b b}}}{m_{B^{*}}-m_{B}}\left|\psi_{B}^{b \bar{q}}(0)\right|^{2} \equiv r_{\Xi_{b b}}\left|\psi_{B}^{b \bar{q}}(0)\right|^{2}, \\
\left|\psi^{s, b b}(0)\right|^{2} & =\frac{4}{3} \frac{m_{\Omega_{b b}^{*}}-m_{\Omega_{b b}}}{m_{B_{s}^{*}}-m_{B_{s}}}\left|\psi_{B_{s}}^{b \bar{s}}(0)\right|^{2} \equiv r_{\Omega_{b b}}\left|\psi_{B_{s}}^{b \bar{s}}(0)\right|^{2} .
\end{aligned}
$$

\section{B. Numerical results}

To compute the decay widths of doubly heavy baryons, we have to specify the values of $\tilde{B}$ and $r_{\mathcal{B}_{Q Q^{\prime}}}$. Since $\tilde{B}=1$ in the valence-quark approximation and since the wave function squared ratio $r_{\mathcal{B}_{Q Q^{\prime}}}$ defined in Eq. (3.17) or Eq. (3.21) is evaluated using the quark model, it is reasonable to assume that the nonrelativistic quark model and the valence-quark approximation are most reliable when the baryon matrix elements are evaluated at a typical hadronic scale $\mu_{\text {had }}$. As shown in [27], the parameters $\tilde{B}$ and $r$ renormalized at two different scales are related via the renormalization group equation to be

$$
\begin{aligned}
\tilde{B}(\mu) r(\mu) & =\tilde{B}\left(\mu_{\mathrm{had}}\right) r\left(\mu_{\mathrm{had}}\right), \\
\tilde{B}(\mu) & =\frac{\tilde{B}\left(\mu_{\mathrm{had}}\right)}{\kappa+\frac{1}{N_{c}}(\kappa-1) \tilde{B}\left(\mu_{\mathrm{had}}\right)},
\end{aligned}
$$

with

$$
\kappa=\left(\frac{\alpha_{s}\left(\mu_{\mathrm{had}}\right)}{\alpha_{s}(\mu)}\right)^{3 N_{c} / 2 \beta_{0}}=\sqrt{\frac{\alpha_{s}\left(\mu_{\mathrm{had}}\right)}{\alpha_{s}(\mu)}}
$$

and $\beta_{0}=\frac{11}{3} N_{c}-\frac{2}{3} n_{f}$. The parameter $\kappa$ takes care of the evolution from $m_{Q}$ to the hadronic scale. We consider the hadronic scale in the range of $\mu_{\text {had }} \sim 0.65-1 \mathrm{GeV}$. Taking the scale $\mu_{\text {had }}=0.9 \mathrm{GeV}$ as an illustration, we obtain $\tilde{B}(\mu)=(0.75,0.67,0.57) \tilde{B}\left(\mu_{\text {had }}\right) \simeq(0.75,0.67,0.57)$ and $r(\mu) \simeq(1.33,1.50,1.74) r\left(\mu_{\text {had }}\right)$. The parameter $\tilde{\beta}$ is treated in a similar way.

We shall discuss the lifetimes of the doubly bottom baryons $\mathcal{B}_{b b}$ first for their simplicity. Following [12], we will use the kinetic $b$ quark mass $m_{b}=4.546 \mathrm{GeV}$ as the calculated inclusive semileptonic $B$ rate to the leading order (LO); using this kinetic mass is very close to the experimental measurement [12]. For numerical calculations, we use the LO Wilson coefficients $c_{1}(\mu)=1.104$ and $c_{2}(\mu)=$ -0.243 evaluated at the scale $\mu=4.546 \mathrm{GeV}, m_{\Xi_{b b}^{*}}-m_{\Xi_{b b}}=$ $35 \mathrm{MeV}$, and $m_{\Omega_{b b}^{*}}-m_{\Omega_{b b}}=30 \mathrm{MeV}$ from [25], the wave function $\left|\psi^{b b}(0)\right|^{2}=0.151 \mathrm{GeV}^{3}$ [26] and the average kinetic energy $T=0.37 \mathrm{GeV}$ [3]. For the decay constants, we use $f_{B}=186 \mathrm{MeV}$ and $f_{B_{s}}=230 \mathrm{MeV}$. For the charm quark mass we use $m_{c}=1.56 \mathrm{GeV}$ fixed from the experimental values for $D^{+}$and $D^{0}$ semileptonic widths [12]. 
TABLE III. Various contributions to the decay rates (in units of $10^{-13} \mathrm{GeV}$ ) of doubly bottom baryons $\mathcal{B}_{b b}$ to order $1 / m_{b}^{4}$ in the $\mathrm{HQE}$ with the hadronic scale $\mu_{\text {had }}=0.825 \mathrm{GeV}$.

\begin{tabular}{lcccccc}
\hline \hline & $\Gamma^{\mathrm{dec}}$ & $\Gamma^{\mathrm{ann}}$ & $\Gamma_{-}^{\mathrm{int}}$ & $\Gamma^{\mathrm{semi}}$ & $\Gamma^{\mathrm{tot}}$ & $\tau\left(10^{-13} s\right)$ \\
\hline$\Xi_{b b}^{0}$ & 5.800 & 1.698 & & 2.090 & 9.587 & 6.87 \\
$\Xi_{b b}^{-}$ & 5.800 & & -0.283 & 2.090 & 7.607 & 8.65 \\
$\Omega_{b b}^{-}$ & 5.802 & & -0.312 & 2.092 & 7.583 & 8.68 \\
\hline \hline
\end{tabular}

The total widths read

$$
\begin{aligned}
& \Gamma\left(\Xi_{b b}^{0}\right)=\Gamma^{\operatorname{dec}}\left(\Xi_{b b}^{0}\right)+\Gamma^{\mathrm{SL}}\left(\Xi_{b b}^{0}\right)+\Gamma_{\text {ann, } b u}^{\mathcal{B}_{b b}}, \\
& \Gamma\left(\Xi_{b b}^{-}\right)=\Gamma^{\operatorname{dec}}\left(\Xi_{b b}^{-}\right)+\Gamma^{\mathrm{SL}}\left(\Xi_{b b}^{-}\right)+\Gamma_{\text {int }_{b b}, b}^{\mathcal{B}_{b b}}, \\
& \Gamma\left(\Omega_{b b}^{-}\right)=\Gamma^{\operatorname{dec}}\left(\Omega_{b b}\right)+\Gamma^{\mathrm{SL}}\left(\Omega_{b b}\right)+\Gamma_{\text {int_ }, b s}^{\mathcal{B}_{b b}}
\end{aligned}
$$

The results of calculations to order $1 / \mathrm{m}_{b}^{4}$ are shown in Table III. The lifetime pattern is $\tau\left(\Omega_{b b}^{-}\right) \sim \tau\left(\Xi_{b b}^{-}\right)>\tau\left(\Xi_{b b}^{0}\right)$. The $\Xi_{b b}^{0}$ baryon is shortest-lived owing to the $W$-exchange contributions, while $\Xi_{b b}^{-}$and $\Omega_{b b}^{-}$have similar lifetimes as they both receive contributions from destructive Pauli interference. We find the lifetime ratio $\tau\left(\Xi_{b b}^{-}\right) / \tau\left(\Xi_{b b}^{0}\right)=$ 1.26 .

For the numerical calculations in the charm-bottom $\mathcal{B}_{b c}$ system, we also need the LO Wilson coefficients $c_{1}(\mu)=1.211$ and $c_{2}(\mu)=-0.430$ at the charm scale $\mu=1.56 \mathrm{GeV}$, and the Wilson coefficients $c_{1}(\mu)=$ 1.172 and $c_{2}(\mu)=-0.366$ at $\mu=2 m_{r}=2.32 \mathrm{GeV}$. We use $m_{\Xi_{b c}^{*}}-m_{\Xi_{b c}}=47 \mathrm{MeV}, m_{\Omega_{b c}^{*}}-m_{\Omega_{b c}}=42 \mathrm{MeV}$ from [25], and the wave function $\left|\psi^{b c}(0)\right|^{2}=0.065 \mathrm{GeV}^{3}$ [26]. The total inclusive rates for the charm-bottom baryons $\mathcal{B}_{b c}$ read

$$
\begin{aligned}
& \Gamma\left(\Xi_{b c}^{+}\right)=\Gamma^{\mathrm{dec}}\left(\Xi_{b c}^{+}\right)+\Gamma^{\mathrm{SL}}\left(\Xi_{b c}^{+}\right)+\Gamma_{\mathrm{ann}, b c}^{\mathcal{B}_{b c}}+\Gamma_{\mathrm{ann}, b u}^{\mathcal{B}_{b c}} \\
& +\Gamma_{\text {int }_{+}, b c}^{\mathcal{B}_{b c}}+\Gamma_{\text {int }_{-}, c u}^{\mathcal{B}_{b c}}+\Gamma_{\text {int }}^{\mathrm{SL}, b c}, \\
& \Gamma\left(\Xi_{b c}^{0}\right)=\Gamma^{\operatorname{dec}}\left(\Xi_{b c}^{0}\right)+\Gamma^{\mathrm{SL}}\left(\Xi_{b c}^{0}\right)+\Gamma_{\mathrm{ann}, b c}^{\mathcal{B}_{b c}}+\Gamma_{\mathrm{ann}, c d}^{\mathcal{B}_{b c}} \\
& +\Gamma_{\text {int }_{+}, b c}^{\mathcal{B}_{b c}}+\Gamma_{\text {int }_{-}, b d}^{\mathcal{B}_{b c}}+\Gamma_{\text {int }}^{\mathrm{SL}, b c}, \\
& \Gamma\left(\Omega_{b c}^{0}\right)=\Gamma^{\operatorname{dec}}\left(\Omega_{b c}^{0}\right)+\Gamma^{\mathrm{SL}}\left(\Omega_{b c}^{0}\right)+\Gamma_{\mathrm{ann}, b c}^{\mathcal{B}_{b c}}+\Gamma_{\mathrm{int}_{+}, b c}^{\mathcal{B}_{b c}} \\
& +\Gamma_{\text {int }_{+}, c s}^{\mathcal{B}_{b c}}+\Gamma_{\text {int_ }, b s^{\mathcal{B}_{b c}}}^{\mathcal{B}_{\text {int }}}+\Gamma_{\mathrm{int}}^{\mathrm{SL}, b c}+\Gamma_{\mathrm{int}}^{\mathrm{SL}, c s} .
\end{aligned}
$$

The results of calculations to order $1 / \mathrm{m}_{b}^{3}$ and $1 / \mathrm{m}_{c}^{3}$ are exhibited in Table IV. The lifetime hierarchy now reads

$$
\mathcal{O}\left(1 / m_{Q}^{3}\right) \Rightarrow \tau\left(\Xi_{b c}^{+}\right)>\tau\left(\Omega_{b c}^{0}\right)>\tau\left(\Xi_{b c}^{0}\right) .
$$

Since the CKM matrix element $V_{c s}$ is much larger than $V_{c b}$ in magnitude so that $m_{c}^{2}\left|V_{c s}\right|^{2} \gg m_{b}^{2}\left|V_{c b}\right|^{2}$, it is obvious that the spectator effects due to $W$-exchange, constructive and destructive Pauli interferences are dominated by the charm quark, namely $\Gamma_{\text {ann, }, c d}^{\mathcal{B}_{b c}}, \Gamma_{\text {int }+, c s}^{\mathcal{B}_{b c}}$, and $\Gamma_{\text {int- }, c u}^{\mathcal{B}_{b c}}$,
TABLE IV. Various contributions to the decay rates (in units of $10^{-12} \mathrm{GeV}$ ) of doubly charm-bottom baryons $\mathcal{B}_{b c}$ to order $1 / \mathrm{m}_{b}^{3}$ and $1 / m_{c}^{3}$ in the HQE with the hadronic scale $\mu_{\text {had }}=0.900 \mathrm{GeV}$.

\begin{tabular}{lccccccc}
\hline \hline & $\Gamma^{\text {dec }}$ & $\Gamma^{\text {ann }}$ & $\Gamma_{+}^{\text {int }}$ & $\Gamma_{-}^{\text {int }}$ & $\Gamma^{\text {semi }}$ & $\Gamma^{\text {tot }}$ & $\tau\left(10^{-13} s\right)$ \\
\hline$\Xi_{b c}^{+}$ & 1.505 & 0.034 & $0.060-0.737$ & 0.351 & 1.212 & 5.43 \\
$\Xi_{b c}^{0}$ & 1.505 & 3.807 & $0.060-0.008$ & 0.351 & 5.714 & 1.15 \\
$\Omega_{b c}^{0}$ & 1.505 & & 1.795 & -0.010 & 0.757 & 4.047 & 1.63 \\
\hline \hline
\end{tabular}

respectively. Therefore, the large $W$-exchange contribution to $\Xi_{b c}^{0}$ through the subprocess $c d \rightarrow u s \rightarrow c d$ and the sizable destructive Pauli interference contribution to $\Xi_{b c}^{+}$ (see Fig. 2) implies a substantial lifetime difference between $\Xi_{b c}^{+}$and $\Xi_{b c}^{0}$. Numerically, we see from Table IV that $\Gamma_{\text {ann, }, c d}^{\mathcal{B}_{b c}}>\Gamma_{\text {int }+, c s}^{\mathcal{B}_{b c}}>\left|\Gamma_{\text {int- }, c u}^{\mathcal{B}_{b c}}\right|$. This explains the lifetime hierarchy Eq. (3.26). Our lifetime pattern for $\mathcal{B}_{b c}$ baryons to order $1 / m_{Q}^{3}$ is different from that predicted in [5,9] (see Table II). Note that $\Omega_{b c}$ has a larger semileptonic rate due to an additional contribution from constructive Pauli interference.

As shown in [12], the heavy quark expansion in $1 / m_{c}$ does not work well for describing the lifetime pattern of singly charmed baryons. Since the charm quark is not heavy enough, it is sensible to consider the subleading $1 / m_{c}$ and $1 / m_{b}$ corrections to spectator effects as depicted in Eqs. (2.28) and (3.14). The numerical results are shown in Table V. By comparing this table with Table IV, we see that the lifetimes of $\Xi_{b c}^{+}$and $\Xi_{b c}^{0}$ become shorter, whereas $\tau\left(\Omega_{b c}^{0}\right)$ becomes the longest one. The lifetime hierarchy to order $1 / m_{Q}^{4}$ in the HQE is modified to $\tau\left(\Omega_{b c}^{0}\right)>\tau\left(\Xi_{b c}^{+}\right)>\tau\left(\Xi_{b c}^{0}\right)$. This is similar to the case of singly charmed baryons where the calculated $\Omega_{c}^{0}$ lifetime becomes entirely unexpected: the shortest-lived $\Omega_{c}^{0}$ to $\mathcal{O}\left(1 / m_{c}^{4}\right)$ turns out to be the longestlived one to $\mathcal{O}\left(1 / m_{c}^{4}\right)$. This is because $\Gamma_{+}^{\text {int }}$ and $\Gamma^{\text {semi }}$ for $\Omega_{b c}^{0}$ are subject to a large cancellation between dimension- 6 and -7 operators. We see from Table V that $\Gamma_{+}^{\text {int }}\left(\Omega_{b c}^{0}\right)$ even becomes negative, while $\Gamma_{-}^{\text {int }}\left(\Xi_{b c}^{+}\right)$becomes positive. This is because the dimension-7 contribution $\Gamma_{+, 7}^{\text {int }}\left(\Omega_{b c}^{0}\right)$ is destructive and its size is so large that it overcomes the dimension-6 one and flips the sign. This implies that the subleading corrections are too large to justify the validity of the HQE.

In order to allow a description of the $1 / m_{c}^{4}$ corrections to $\Gamma\left(\mathcal{B}_{b c}^{0}\right)$ within the realm of perturbation theory, we follow

TABLE V. Various contributions to the decay rates (in units of $10^{-12} \mathrm{GeV}$ ) of doubly charm-bottom baryons $\mathcal{B}_{b c}$ to order $1 / \mathrm{m}_{Q}^{4}$ in the HQE with the hadronic scale $\mu_{\text {had }}=0.900 \mathrm{GeV}$.

\begin{tabular}{lcrrrrrc}
\hline \hline & $\Gamma^{\text {dec }}$ & \multicolumn{1}{c}{$\Gamma^{\text {ann }}$} & \multicolumn{1}{c}{$\Gamma_{+}^{\text {int }}$} & \multicolumn{1}{c}{$\Gamma_{-}^{\text {int }}$} & $\Gamma^{\text {semi }}$ & $\Gamma^{\text {tot }}$ & $\tau\left(10^{-13} s\right)$ \\
\hline$\Xi_{b c}^{+}$ & 1.505 & -0.044 & 0.014 & 0.028 & 0.346 & 1.848 & 3.56 \\
$\Xi_{b c}^{0}$ & 1.505 & 5.911 & 0.014 & -0.005 & 0.346 & 7.770 & 0.85 \\
$\Omega_{b c}^{0}$ & 1.505 & -0.086 & -0.759 & -0.005 & 0.165 & 0.819 & 8.03 \\
\hline \hline
\end{tabular}


TABLE VI. Various contributions to the decay rates (in units of $10^{-12} \mathrm{GeV}$ ) of the $\Omega_{b c}^{0}$ after including subleading $1 / m_{c}$ corrections to spectator effects. However, the dimension-7 contributions $\Gamma_{\text {int }+, 7}^{c s}\left(\Omega_{b c}^{0}\right), \Gamma_{\text {int,7 }}^{\mathrm{SL}, c s}\left(\Omega_{b c}^{0}\right), \Gamma_{\text {int }-7,7}^{c u}\left(\Xi_{b c}^{+}\right)$, and $\Gamma_{\text {ann,7 }}^{c d}\left(\Xi_{b c}^{0}\right)$ are multiplied by a factor of $(1-\alpha)$ with $\alpha$ varying from 0 to 1 .

\begin{tabular}{lcccccccc}
\hline \hline$\alpha$ & $\Gamma^{\text {dec }}$ & $\Gamma^{\text {ann }}$ & $\Gamma_{+}^{\text {int }}$ & $\Gamma_{-}^{\text {int }}$ & $\Gamma^{\text {semi }}$ & $\Gamma^{\text {tot }}$ & $\tau\left(\Omega_{b c}^{0}\right) \times 10^{13}$ & $\Gamma_{-}^{\text {int }}\left(\Xi_{b c}^{+}\right)$ \\
\hline 0 & 1.505 & -0.086 & -0.759 & -0.005 & 0.165 & 0.820 & 8.03 & 0.019 \\
0.31 & 1.505 & -0.086 & 0.019 & -0.005 & 0.347 & 1.780 & 3.70 & -0.209 \\
0.45 & 1.505 & -0.086 & 0.370 & -0.005 & 0.429 & 2.213 & 2.97 & -0.316 \\
1 & 1.505 & -0.086 & 1.750 & -0.005 & 0.752 & 3.916 & 1.68 & -0.737 \\
\hline \hline
\end{tabular}

TABLE VII. Predicted lifetimes (in units of $10^{-13}$ s) of $\mathcal{B}_{b b}$ and $\mathcal{B}_{b c}$ baryons in this work.

\begin{tabular}{lllccc}
\hline \hline$\Xi_{b b}^{0}$ & $\Xi_{b b}^{-}$ & $\Omega_{b b}^{-}$ & $\Xi_{b c}^{+}$ & $\Xi_{b c}^{0}$ & $\Omega_{b c}^{0}$ \\
\hline 6.87 & 8.65 & 8.68 & $4.09-6.07$ & $0.93-1.18$ & $1.68-3.70$ \\
\hline \hline
\end{tabular}

[12] to introduce a parameter $\alpha$ so that $\Gamma_{\mathrm{int}+, 7}^{c s}\left(\Omega_{b c}^{0}\right)$, $\Gamma_{\text {int }, 7}^{\mathrm{SL}, c s}\left(\Omega_{b c}^{0}\right), \Gamma_{\text {int }-, 7}^{c u}\left(\Xi_{b c}^{+}\right)$, and $\Gamma_{\text {ann }, 7}^{c d}\left(\Xi_{b c}^{0}\right)$ are multiplied by a factor of $(1-\alpha)$; that is, $\alpha$ describes the degree of suppression. In Table VI we show the variation of the $\Omega_{b c}^{0}$ lifetime with $\alpha$. At $\alpha=0.31, \Gamma_{+}^{\text {int }}\left(\Omega_{b c}^{0}\right)$ starts to become positive and $\tau\left(\Omega_{b c}^{0}\right)=3.64 \times 10^{-13} \mathrm{~s}$. Since we do not know what the value of $\alpha$ is, we can only conjecture that it lies in $0.31<\alpha<1$ and the $\Omega_{b c}^{0}$ lifetime lies in the range

$$
1.68 \times 10^{-13} s<\tau\left(\Omega_{b c}^{0}\right)<3.70 \times 10^{-13} s .
$$

Likewise,

$$
\begin{aligned}
& 4.09 \times 10^{-13} s<\tau\left(\Xi_{b c}^{+}\right)<6.07 \times 10^{-13} s, \\
& 0.93 \times 10^{-13} s<\tau\left(\Xi_{b c}^{0}\right)<1.18 \times 10^{-13} s,
\end{aligned}
$$

with the lifetime pattern $\tau\left(\Xi_{b c}^{+}\right)>\tau\left(\Omega_{b c}^{0}\right)>\tau\left(\Xi_{b c}^{0}\right)$. The predicted lifetimes of $\mathcal{B}_{b b}$ and $\mathcal{B}_{b c}$ baryons in this work are summarized in Table VII.

\section{Comparison with other works}

Comparing Table VII with Table II, we see that our lifetime pattern $\tau\left(\Omega_{b b}^{-}\right) \sim \tau\left(\Xi_{b b}^{-}\right)>\tau\left(\Xi_{b b}^{0}\right)$ for $\mathcal{B}_{b b}$ baryons is different from the one $\tau\left(\Xi_{b b}^{-}\right) \approx \tau\left(\Xi_{b b}^{0}\right)$ in [7] and the one $\tau\left(\Omega_{b b}^{-}\right) \approx \tau\left(\Xi_{b b}^{-}\right) \sim \tau\left(\Xi_{b b}^{0}\right)$ in $[5,9,10]$. The $\Xi_{b b}^{0}$ baryon is shortest-lived owing to the $W$-exchange contributions, while $\Xi_{b b}^{-}$and $\Omega_{b b}^{-}$have similar lifetimes as they both receive contributions from destructive Pauli interference. We find the lifetime ratio $\tau\left(\Xi_{b b}^{-}\right) / \tau\left(\Xi_{b b}^{0}\right)=1.26$, while it is predicted to be of order unity in $[5,7,9,10]$ (see Table II) due to the smallness of the $W$-exchange in $\Xi_{b b}^{0}$ and destructive Pauli interference in $\Xi_{b b}^{-}$. For example, the ratio of $\Gamma^{\mathrm{ann}}\left(\Xi_{b b}^{0}\right) / \Gamma^{\mathrm{dec}}$ is calculated to be only one percent (see Table III of [9]), whereas it is of order 0.3 in our case (see Table III).
For $\mathcal{B}_{b c}$ baryons, our lifetime hierarchy $\tau\left(\Xi_{b c}^{+}\right)>$ $\tau\left(\Omega_{b c}^{0}\right)>\tau\left(\Xi_{b c}^{0}\right)$ differs from that of $[5,9,10]$ in which one has $\tau\left(\Xi_{b c}^{+}\right)>\tau\left(\Xi_{b c}^{0}\right)>\tau\left(\Omega_{b c}^{0}\right)$. Since the $b c$ diquark is treated to be a scalar one with $S_{b c}=0$ by the authors of $[5,9,10]$, their $\mathcal{B}_{b c}$ quark matrix elements of four-quark operators and chromomagnetic interactions differ from ours. However, irrespective of the way of treating the $\mathcal{B}_{b c}$ quark matrix elements, an inspection of Fig. 2 leads to the pattern $\Gamma^{\mathrm{ann}}\left(\Xi_{b c}^{0}\right)>\Gamma^{\mathrm{ann}}\left(\Xi_{b c}^{+}\right)>\Gamma^{\mathrm{ann}}\left(\Omega_{b c}^{0}\right)$. All three $\mathcal{B}_{b c}$ baryons receive a common $W$-exchange contribution through the subprocess $b c \rightarrow c s \rightarrow b c$, but $\Xi_{b c}^{+}$gets an additional $W$-exchange between the $b$ and $u$ quarks, while $\Xi_{b c}^{0}$ receives a large $W$-exchange contribution through the subprocess $c d \rightarrow s u \rightarrow c d$. Hence, it is not clear to us why $\Omega_{b c}$ has the largest $W$-exchange in $[5,9,10]$ (see e.g., Table II of [9]).

As to the Pauli interference, the destructive contribution to $\Xi_{b c}^{+}$should be larger than the constructive one in magnitude due to the large CKM matrix element $V_{c s}$. As a consequence, the net Pauli interference in $\Xi_{b c}^{+}$should be negative, while it was calculated to be positive in $[5,9,10]$.

\section{CONCLUSIONS}

In this work we have analyzed the lifetimes of the doubly heavy baryons $\mathcal{B}_{b b}$ and $\mathcal{B}_{b c}$ within the framework of the heavy quark expansion. It is well known that the lifetime differences stem from spectator effects such as $W$-exchange and Pauli interference. We rely on the quark model to evaluate the hadronic matrix elements of dimension-6 and -7 four-quark operators responsible for spectator effects.

The main results of our analysis are as follows.

(i) Special attention is paid to the doubly heavy baryon matrix elements of dimension- 3 and -5 operators which are different from the ones of singly heavy baryons. The doubly heavy baryon matrix element of the $\sigma \cdot G$ operator receives three distinct contributions: the interaction of the heavy quark with the chromomagnetic field produced from the light quark and from the other heavy quark, and the so-called Darwin term in which the heavy quark interacts with the chromoelectric field. For $\mathcal{B}_{b c}$ baryons, the first contribution is proportional to the hyperfine mass splitting of $\mathcal{B}_{b c}$. However, it vanishes if the $b c$ 
diquark is wrongly assigned to be of the scalar type as often assumed in the previous studies.

(ii) For doubly bottom baryons, the lifetime pattern is $\tau\left(\Omega_{b b}^{-}\right) \sim \tau\left(\Xi_{b b}^{-}\right)>\tau\left(\Xi_{b b}^{0}\right)$. The $\Xi_{b b}^{0}$ baryon is shortestlived owing to the $W$-exchange contributions, while $\Xi_{b b}^{-}$and $\Omega_{b b}^{-}$have similar lifetimes as they both receive contributions from destructive Pauli interference. We find the lifetime ratio $\tau\left(\Xi_{b b}^{-}\right) / \tau\left(\Xi_{b b}^{0}\right)=$ 1.26 .

(iii) The study of $\mathcal{B}_{b c}$ lifetimes is more complicated than the $\mathcal{B}_{b b}$ case for several reasons. First, besides the spectator effects due to each heavy quark $b$ or $c$, there also exist $W$-exchange and Pauli interference in which both $b$ and $c$ quarks get involved. Second, care must be taken when considering the heavy quark expansion for the charm quark.

(iv) The large $W$-exchange contribution to $\Xi_{b c}^{0}$ through the subprocess $c d \rightarrow u s \rightarrow c d$ and the sizable destructive Pauli interference contribution to $\Xi_{b c}^{+}$imply a substantial lifetime difference between $\Xi_{b c}^{+}$and $\Xi_{b c}^{0}$.

(v) In the presence of subleading $1 / m_{c}$ and $1 / m_{b}$ corrections to the spectator effects, we find that
$\tau\left(\Omega_{b c}^{0}\right)$ becomes longest-lived. This is because $\Gamma_{+}^{\text {int }}$ and $\Gamma^{\mathrm{semi}}$ for $\Omega_{b c}^{0}$ are subject to large cancellation between dimension- 6 and -7 operators. This implies that the subleading corrections are too large to justify the validity of the HQE. Demanding that $\Gamma_{\text {int }+}^{c s}\left(\Omega_{b c}^{0}\right), \Gamma_{\text {int }}^{S \mathrm{~L}, c s}\left(\Omega_{b c}^{0}\right)$ be positive and $\Gamma_{\text {int- }}^{c u}\left(\Xi_{b c}^{+}\right)$be negative, we conjecture that $1.68 \times 10^{-13} s<\tau\left(\Omega_{b c}^{0}\right)<$ $3.70 \times 10^{-13} \mathrm{~s}, 4.09 \times 10^{-13} s<\tau\left(\Xi_{b c}^{+}\right)<6.07 \times 10^{-13} \mathrm{~s}$, and $0.93 \times 10^{-13} s<\tau\left(\Xi_{b c}^{0}\right)<1.18 \times 10^{-13} \mathrm{~s}$.

(vi) The lifetime hierarchy in the $\mathcal{B}_{b c}$ system is expected to be $\tau\left(\Xi_{b c}^{+}\right)>\tau\left(\Omega_{b c}^{0}\right)>\tau\left(\Xi_{b c}^{0}\right)$. We have compared our work with others.

\section{ACKNOWLEDGMENTS}

This research was supported in part by the Ministry of Science and Technology of R.O.C. under Grant No. 1072119-M-001-034. F. Xu was supported by NSFC under Grant No. 11605076 as well as the Fundamental Research Funds for the Central Universities in China under the Grant No. 21616309.
[1] R. Aaij et al. (LHCb Collaboration), Observation of the Doubly Charmed Baryon $\Xi_{c c}^{++}$, Phys. Rev. Lett. 119, 112001 (2017).

[2] R. Aaij et al. (LHCb Collaboration), First Measurement of the Lifetime of the Doubly Charmed Baryon $\Xi_{c c}^{++}$, Phys. Rev. Lett. 121, 052002 (2018).

[3] V. V. Kiselev, A. K. Likhoded, and A. I. Onishchenko, Lifetimes of doubly charmed baryons: $\Xi_{c c}^{+}$and $\Xi_{c c}^{++}$, Phys. Rev. D 60, 014007 (1999).

[4] B. Guberina, B. Melić, and H. Štefančić, Inclusive decays and lifetimes of doubly charmed baryons, Eur. Phys. J. C 9, 213 (1999); 13, 551(E) (2000).

[5] V. V. Kiselev and A. K. Likhoded, Baryons with two heavy quarks, Usp. Fiz. Nauk 172, 497 (2002) [Phys. Usp. 45, 455 (2002)].

[6] C. H. Chang, T. Li, X. Q. Li, and Y. M. Wang, Lifetime of doubly charmed baryons, Commun. Theor. Phys. 49, 993 (2008).

[7] M. Karliner and J. L. Rosner, Baryons with two heavy quarks: Masses, production, decays, and detection, Phys. Rev. D 90, 094007 (2014).

[8] H. Y. Cheng and Y. L. Shi, Lifetimes of doubly charmed baryons, Phys. Rev. D 98, 113005 (2018).

[9] A. V. Berezhnoy, A. K. Likhoded, and A. V. Luchinsky, Doubly heavy baryons at the LHC, Phys. Rev. D 98, 113004 (2018).

[10] A. K. Likhoded and A. I. Onishchenko, Lifetimes of doubly heavy baryons, arXiv:hep-ph/9912425.
[11] V. V. Kiselev, A. K. Likhoded, and A. I. Onishchenko, Lifetimes of $\Xi_{b c}^{+}$and $\Xi_{b c}^{0}$ baryons, Eur. Phys. J. C 16, 461 (2000).

[12] H. Y. Cheng, Phenomenological study of heavy hadron lifetimes, J. High Energy Phys. 11 (2018) 014.

[13] R. Aaij et al. (LHCb Collaboration), Measurement of the $\Omega_{c}^{0}$ Baryon Lifetime, Phys. Rev. Lett. 121, 092003 (2018).

[14] M. Tanabashi et al. (Particle Data Group), Review of Particle Physics, Phys. Rev. D 98, 030001 (2018).

[15] Z. S. Brown, W. Detmold, S. Meinel, and K. Orginos, Charmed bottom baryon spectroscopy from lattice QCD, Phys. Rev. D 90, 094507 (2014).

[16] L. Tang, X.-H. Yuan, C.-F. Qiao, and X.-Q. Li, Study of doubly heavy baryon spectrum via QCD sum rules, Commun. Theor. Phys. 57, 435 (2012).

[17] I. I. Bigi, N. G. Uraltsev, and A. I. Vainshtein, Nonperturbative corrections to inclusive beauty and charm decays: QCD versus phenomenological models, Phys. Lett. B 293, 430 (1992); 297, 477(E) (1992).

[18] B. Blok and M. A. Shifman, The Rule of discarding $1 / N_{c}$ in inclusive weak decays. 1., Nucl. Phys. B399, 441 (1993); The Rule of discarding $1 / N_{c}$ in inclusive weak decays. 2 ., Nucl. Phys. B399, 459 (1993); Lifetimes of charmed hadrons revisited. Facts and fancy, in Proceedings of the Third Workshop on the Physics at a Tau-Charm Factory, Marbella, Spain, edited by J. Kirkby and R. Kirkby (Editions Frontieres, Gif-sur-Yvette, France, 1994).

[19] A. De Rujula, H. Georgi, and S. L. Glashow, Hadron masses in a gauge theory, Phys. Rev. D 12, 147 (1975). 
[20] M. Beneke and G. Buchalla, The $B_{c}$ meson lifetime, Phys. Rev. D 53, 4991 (1996).

[21] M. B. Voloshin, Spectator effects in semileptonic decay of charmed baryons, Phys. Lett. B 385, 369 (1996).

[22] F. Gabbiani, A. I. Onishchenko, and A. A. Petrov, $\Lambda_{b}$ lifetime puzzle in heavy quark expansion, Phys. Rev. D 68, 114006 (2003).

[23] F. Gabbiani, A. I. Onishchenko, and A. A. Petrov, Spectator effects and lifetimes of heavy hadrons, Phys. Rev. D 70, 094031 (2004).
[24] A. Lenz and T. Rauh, $D$-meson lifetimes within the heavy quark expansion, Phys. Rev. D 88, 034004 (2013).

[25] D. Ebert, R. N. Faustov, V. O. Galkin, and A. P. Martynenko, Properties of doubly heavy baryons in the relativistic quark model, Yad. Fiz. 68, 817 (2005) [Phys. At. Nucl. 68, 784 (2005)].

[26] S. P. Baranov, On the production of doubly flavored baryons in pp, ep and $\gamma \gamma$ collisions, Phys. Rev. D 54, 3228 (1996).

[27] M. Neubert and C. T. Sachrajda, Spectator effects in inclusive decays of beauty hadrons, Nucl. Phys. B483, 339 (1997). 